\title{
Respuesta termográfica de bloques de concreto para uso no estructural preparados con ceniza industrial
}

\author{
Cristian David Ballesteros Giraldo ${ }^{1}$ \\ María Fernanda Serrano GuZmán ${ }^{2}$ \\ Diego Darío PÉrez Ruiz ${ }^{3}$
}

RECIBIDO: 30/08/2018 ACEPTADO: 25/04/2019

\begin{abstract}
RESUMEN
La ceniza es un residuo proveniente de diferentes actividades comerciales. En el caso de la industria del papel, este resto que proviene de la combustión del carbón empleado en el proceso productivo representa un problema en su disposición. Esa es la razón por la cual se ha evaluado su aprovechamiento en la producción de bloques de concreto. Este estudio tiene como objetivo comparar la respuesta termográfica de bloques de concreto para aplicaciones no estructurales que han sido preparados con esta ceniza y manufacturados de forma industrial. Adicionalmente, se compara el comportamiento mecánico de los bloques cuando se sustituye porcentualmente la ceniza por el cemento. Asimismo, se hicieron sustituciones del 10, 20, 30 y $40 \%$ de ceniza por cemento en bloques preparados según control de calidad de una fábrica de bloques. Ensayos especializados, tales como la difracción de rayos $X$ y la microscopía, demostraron la ausencia de sustancias tóxicas en la ceniza. El estudio reveló que la capacidad de absorción térmica aumenta a medida que aumenta la cantidad de ceniza.
\end{abstract}

Palabras-claves: Bloques; ceniza; residuos industriales; aislamiento térmico; resistencia.

\section{INTRODUCCIÓN}

La construcción es un sector económico que dinamiza diferentes sectores productivos y también exige el consumo de diferentes materias primas, equipo y recurso humano (Cámara Colombiana de la Construcción y Coordenada Urbana, 2014). En general, esta industria genera importantes ingresos, pero también produce muchos residuos. No obstante, otras industrias, como las de fabricación de papel, también generan residuos, tipo ceniza, cuya producción puede alcanzar hasta dos toneladas por mes (Vidal, 2016). El exceso de producción de residuos y los inconvenientes causados por la falta de espacio para la disposición de los mismos, así como la necesidad de mantener un consumo sostenible de las materias primas, ha incentivado estudios en los cuales se evalúa el efecto de la adición de residuos industriales, como la ceniza, en la producción de cemento o concreto. Por esta razón, la ceniza que proviene de diversas fuentes como la incineración de la cáscara de arroz, la desorción térmica de lodos provenientes del proceso de extracción de hidrocarburos, la incineración de combustibles, de centrales térmicas, entre otras, ha sido evaluada para darle otro uso, obteniéndose variados resultados.

Por ejemplo, la cáscara de arroz en proporciones de 5 a $20 \%$ arrojó mezclas de concreto con baja permeabilidad por la reducción de los poros, situación que mejora la resistencia de estas mezclas al ataque de agentes agresivos (Azevedo et al., 2001). Posteriormente, en un estudio de Giaccio et al. (2007) se empleó la sustitución del $10 \%$ de cemento por ceniza y se obtuvieron mezclas de concreto de alta resistencia (80 MPa) con un buen comportamiento de absorción superficial. Otros estudios reportan que dosificaciones del $20 \%$ de ceniza volante y del $20 \%$ de ceniza proveniente del arroz, luego de 56 días de curado, permiten desarrollar concretos con resistencias de hasta $130 \mathrm{MPa}$

1 Magister en Ingeniería Civil por la Pontificia Universidad Javeriana (Cali, Colombia). Actualmente, es consultor independiente.

E-mail: davidballesteros1@hotmail.com

2 Doctora en Ingeniería Civil de la Universidad de Puerto Rico en Mayagüez, Puerto Rico. Actualmente, es profesora de Ingeniería Civil en la Pontificia Universidad Javeriana de Cali, Colombia.

E-mail: maria.serrano@javerianacali.edu.co

Orcid: http://orcid.org/0000-0002-7366-6597

3 Doctor en Ingeniería Civil de la Universidad de Texas en Arlington, Estados Unidos. Actualmente, es profesor de Ingeniería Civil en la Pontificia Universidad Javeriana de Cali, Colombia. E-mail: ddperez@javerianacali.edu.co 
(Le y Ludwig, 2016). En el caso de la ceniza proveniente de procesos de desorción térmica de lodos de locaciones petroleras, puede acelerar el tiempo de fraguado inicial cuando es añadida en porcentajes menores a $15 \%$, aunque se aprecia una reducción de la resistencia a la compresión hasta de $12 \%$ con relación a las mezclas testigo (Serrano et al., 2013). Por otro lado, con la ceniza proveniente de la incineración de combustibles se han producido mezclas con resistencias a compresión hasta de $125 \mathrm{MPa}$ cuando se adiciona en porcentajes del 25 y $55 \%$ (Xu et al., 2003). Con las cenizas volcánicas añadidas en porcentajes del $50 \%$, las mezclas han presentado una resistencia a compresión en promedio de $15 \mathrm{MPa}$ (Hossain y Lachemi, 2010). Finalmente, en cuanto a la ceniza producto de la combustión de carbón pulverizado en plantas de energía, cuando es añadida como sustitución del $10 \%$ del cemento, produce comportamientos eficaces en la mejora de las propiedades mecánicas del concreto (Asi y Abdullah, 2005), pudiendo alcanzar en algunos casos resistencias de hasta $51 \mathrm{MPa}$ (Nuruddin et al., 2011), además que se mejora la durabilidad del concreto expuesto a medios salinos (Chousidis et al., 2015).

Como bien afirman Topcu et al. (2008), los orígenes de la ceniza son variados, así como los usos en la producción de mortero y concreto (Teixeira et al., 2016) para la fabricación de bloques de concreto para uso no estructural como gres porcelánico (Delgado et al., 2015), en concretos y morteros modificados (Serrano et al., 2012; Serrano et al., 2013), así como también como material empleado en soluciones de geotecnia y suelos y en otros usos de tipo agrícola (Slim et al., 2016) o en andenes (Bouzoubaâ et al., 2011). Asimismo, como la ceniza es empleada como sustitución del cemento, las mezclas producidas son más económicas y, en la mayoría de estudios realizados, han demostrado aumento de la resistencia y la durabilidad de las mezclas, llegando a recomendarse su uso en concretos de alto desempeño (Bui et al., 2005).

En este contexto, el objetivo de este trabajo es presentar la respuesta termográfica al incluir un residuo de la industria del papel en la fabricación de bloques de concreto conforme al sistema productivo de una empresa colombiana y evaluar la respuesta mecánica a compresión de los bloques producidos frente a los estándares de dicha empresa. Este tipo de mampuesto abre el espacio para una alianza entre los productores de bloques y de papel por cuanto los resultados de esta investigación indican que la adición de ceniza no afectó la resistencia a compresión del bloque producido y mejoró su respuesta a los cambios de temperatura.

\section{METODOLOGÍA}

El análisis de la respuesta térmica de bloques se hizo en dos etapas: se inició con el análisis de la ceniza suministrada por la fábrica de papel y se finalizó con el análisis de la respuesta térmica de los bloques.

\section{Caracterización microscópica de la ceniza}

Se utilizó un microscopio electrónico de barrido JEOL modelo JSM 6490 LV y se revisaron los cambios de composición química a través de las imágenes mediante un voltaje de aceleración de $15 \mathrm{kV}$. Adicionalmente, se efectuaron microanálisis químicos de las muestras sobre varios puntos o áreas de inspección. Para ello, se utilizó una sonda EDS de Oxford Instrument Modelo INCAPentaFETx3.

\section{Ensayo difracción de rayos $X$}

Para los análisis con rayos $\mathrm{X}$, se empleó un equipo de alta resolución (X'Pert-MRD PANalytical) y los datos fueron analizados empleando el software Xpert Data. La caracterización de la muestra se realizó comparando las reflexiones obtenidas con las reportadas en la base de datos PDF2-Release-2009 International Centre for Diffraction Data (ICDD) para materiales puros y policristalinos.

\section{Procedimiento para la fabricación de los blo- ques estudiados}

Cada fabricante de bloques emplea una dosificación particular para la preparación de estos. Dicha dosificación puede variar entre empresas productoras de bloques, señala Fernandes (2015), por cuanto los materiales se acondicionan en una misma línea de producción, según las condiciones del material, estado de la maquinaria y otros factores que afectan la calidad de las piezas (Buitrago, 2015). Por ello, en esta investigación se utilizó un procedimiento industrializado para la producción de treinta bloques típicos del Valle del Cauca (Colombia), dosificación a la cual se le hicieron ajustes para preparar bloques con sustituciones del 10, 20, 30 y $40 \%$ de cemento por ceniza, según las proporciones indicadas en la tabla 1. Adicionalmente, se realizó el ensayo de granulometría para agregados finos siguiendo la norma ASTM C-136.

Las etapas de producción de los bloques estudiados se resumen así:

- Se revisó el estado de la maquinaria y de las materias primas.

- Se estableció la textura requerida para bloques de uso no estructural. 
- Se revisó la cohesión de la mezcla para garantizar que el bloque permaneciera íntegro durante su fabricación.

- Se compactó el bloque y se verificó si tenía consistencia dura (con la prueba del «dedo duro») y si, al verterle agua en la superficie, esta no pasaba libremente a través del material. Se almacenaron los bloques en el cuarto de curado.

- Se llevó a cabo el control de calidad de los bloques según las normas técnicas de calidad del Instituto Colombiano de Normas Técnicas NTC 4205-1, 4205-2 y 4205-3, con el fin de determinar el peso, la absorción total, la densidad, el volumen neto y el área neta.

- Se procedió a realizar el ensayo de compresión de las unidades secas, teniendo en cuenta la dirección en que estarían puestos en servicio los bloques; en este caso, con los huecos verticales (norma NTC-ISO 7500-1 para clase 1).

\section{Pruebas termográficas}

La cámara termográfica debe mantenerse a la misma distancia focal $(0,70 \mathrm{~m})$, perpendicular a la cara del espécimen monitoreada y conservando la misma altura del trípode $(0,30 \mathrm{~m})$. Esta cámara resalta las variaciones de temperatura y los puntos calientes en tiempo real y permite combinar la imagen de luz visible con una imagen térmica procedente del objeto de estudio (Dirección General de Industria, Energía y Minas de la Comunidad de Madrid y la Fundación de la Energía de la Comunidad de Madrid, 2011). Se evaluó la respuesta de temperatura de los bloques previamente expuestos tres días a radiación solar. Adicionalmente, con un sensor de temperatura y humedad se tomaron lecturas de estos parámetros con el fin de incorporar estos datos para la calibración y procesamiento de datos con la cámara.

\section{RESULTADOS}

La arena y el polvillo de cantera presentaron un módulo de finura de 2,43 y 2,78 , respectivamente, por lo que ambos materiales se pueden clasificar como arena con alta presencia de finos. En cuanto a la ceniza, el ensayo realizado con el microscopio electrónico de barrido permitió identificar su composición química (ver tabla 2 ), según resultados obtenidos en los siete puntos señalados en la figura 1, que muestra el detalle microscópico de la ceniza con magnificación de la superficie de cincuenta veces. Los resultados de la composición química indican la presencia de carbono, oxígeno y silicio, con valores promedio de concentración de 48.2, 24.5 y $11.9 \%$, respectivamente, lo que sugiere que la ceniza podría ser utilizada en la producción de concreto.

Tabla 1. Matriz experimental.

\begin{tabular}{|l|c|c|c|c|c|c|}
\hline \multicolumn{1}{|c|}{ Bloque } & Cemento kg & ${\text { Polvillo } \mathbf{~}^{\mathbf{3}}}^{\text {Prena } \mathbf{~}^{3}}$ & Agua I & Aditivo cc & Ceniza kg \\
\hline Testigo & 50 & 0,231 & 0,099 & 18,00 & 200 & 0,00 \\
\hline Sustitución 10\% & 45 & 0,231 & 0,099 & 20,00 & 200 & 5,00 \\
\hline Sustitución 20\% & 40 & 0,231 & 0,099 & 22,00 & 200 & 10,00 \\
\hline Sustitución 30\% & 35 & 0,231 & 0,099 & 25,50 & 200 & 15,00 \\
\hline Sustitución 40\% & 30 & 0,231 & 0,099 & 25,50 & 200 & 20,00 \\
\hline
\end{tabular}

Fuente: basado de Ballesteros (2016).

Tabla 2. Composición química de la ceniza (cantidades en porcentaje).

\begin{tabular}{|l|l|l|l|l|l|l|l|l|l|}
\hline \multicolumn{1}{|c|}{ Espectro } & \multicolumn{1}{c|}{$\mathbf{C}$} & \multicolumn{1}{c|}{$\mathbf{O}$} & $\mathbf{N a}$ & $\mathbf{A l}$ & \multicolumn{1}{c|}{$\mathbf{S i}$} & $\mathbf{K}$ & $\mathbf{C a}$ & $\mathbf{T i}$ & $\mathbf{F e}$ \\
\hline 1 & 11,64 & 36,75 & 2,87 & 19,96 & 23,27 & & & 2,23 & 3,29 \\
\hline 2 & 85,81 & 11,01 & & 1,57 & 1,62 & & & & \\
\hline 3 & 63,55 & 19,20 & & 3,09 & 11,85 & & 2,31 & & \\
\hline 4 & 25,73 & 39,15 & 1,72 & 3,92 & 28,33 & 1,14 & & & \\
\hline 5 & 15,48 & 16,96 & & 5,31 & 8,84 & & 0,88 & & 52,53 \\
\hline 6 & 76,96 & 18,34 & & 2,39 & 2,31 & & & & \\
\hline 7 & 58,20 & 30,20 & & 4,70 & 6,90 & & & & \\
\hline Promedio & 48,20 & 24,52 & 2,30 & 5,85 & 11,87 & 1,14 & 1,60 & 2,23 & 27,91 \\
\hline Desviación estándar & 30,20 & 10,80 & 0,80 & 6,40 & 10,30 & N. A. & 1,00 & N. A. & 34,80 \\
\hline Coeficiente de variación & 62,70 & 44,00 & 34,80 & 109,40 & 86,80 & N. A. & 62,50 & N. A. & 124,70 \\
\hline
\end{tabular}

Fuente: basado de Ballesteros (2016). 
Los resultados del análisis por difracción de rayos $\mathrm{X}$ muestran las fases (códigos 98-010-7202 y 98008-0148) presentes en el material (ver figuras 2 y 3) identificadas en la base de datos consultada como óxido de silicio en estructura cristalográfica hexagonal y óxido de silicio de aluminio en estructura cristalográfica ortorrómbico, respectivamente. Estos resultados sugirieron la posibilidad de usar esta ceniza en la fabricación de bloques.
Con relación a la absorción y densidad de los bloques de concreto, según la Norma Técnica Colombiana NTC 4076, los bloques con ceniza cumplieron lo establecido para concretos con densidades de $2000 \mathrm{~kg} / \mathrm{m}^{3}$ o más, que deben tener un porcentaje de absorción total no superior al 12\% (ver tabla 3).

Los especímenes sin ceniza, así como los especímenes con las diferentes adiciones, presentaron

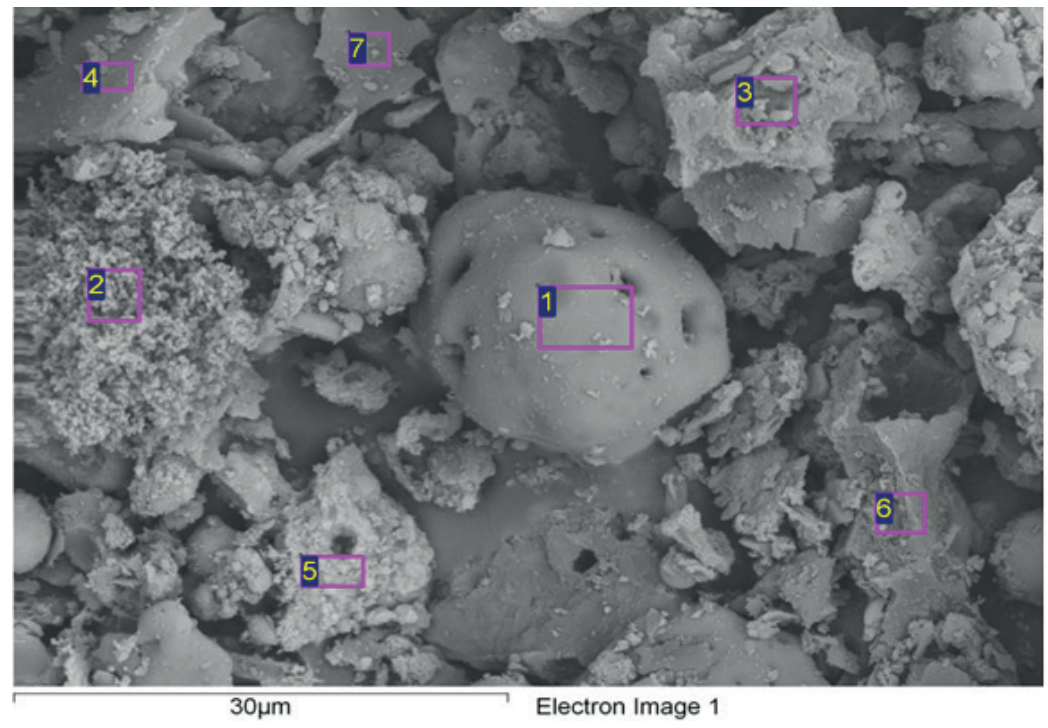

Figura 1. Detalle microscópico de la ceniza.

Fuente: Informe del Laboratorio de Difracción de Rayos X de la Universidad del Valle, tomado de Ballesteros (2016).

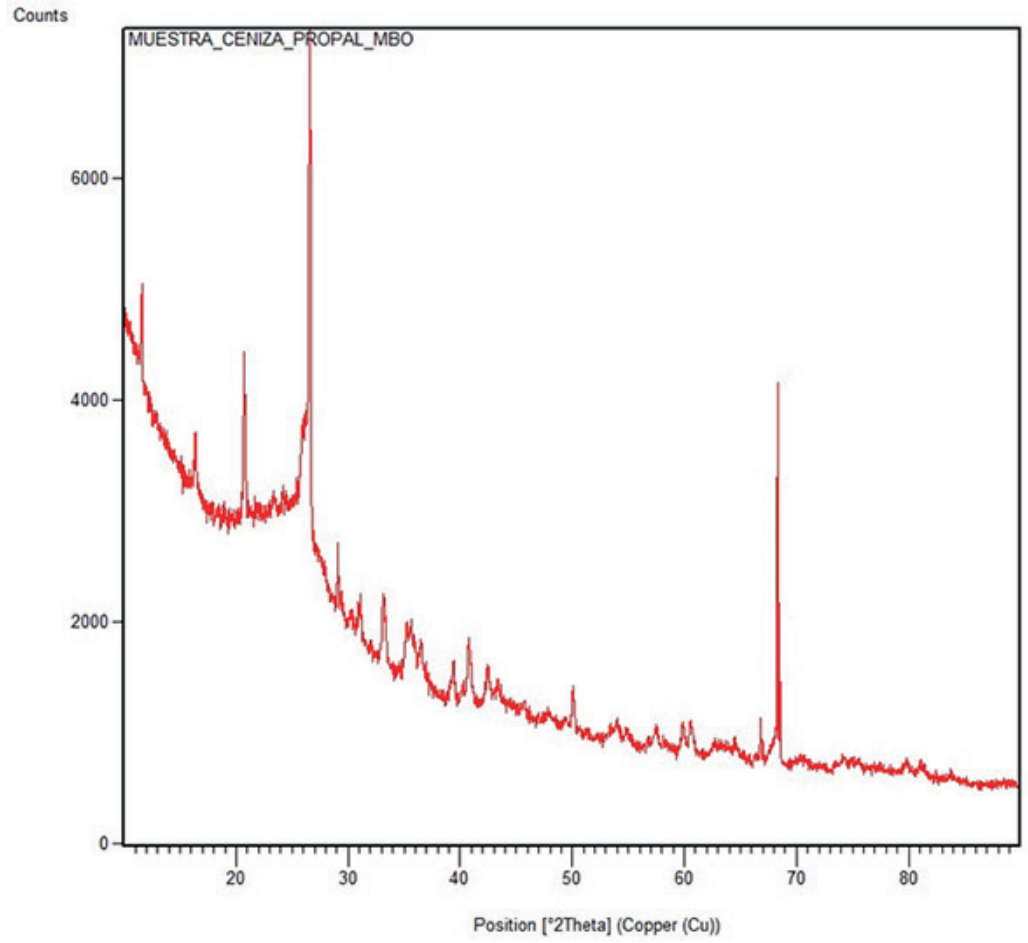

Figura 2. Difractograma de la muestra de ceniza.

Fuente: Informe del Laboratorio de Difracción de Rayos X de la Universidad del Valle, tomado de Ballesteros (2016). 
a los 28 días resistencias mayores o iguales a 10 $\mathrm{MPa}$, que es el valor de la resistencia de los bloques para uso no estructural que se ofrecen en el mercado (ver figura 4).
En general, la resistencia mecánica de los bloques preparados superó la resistencia mecánica de los bloques ofrecidos normalmente en el mercado (tabla 4).

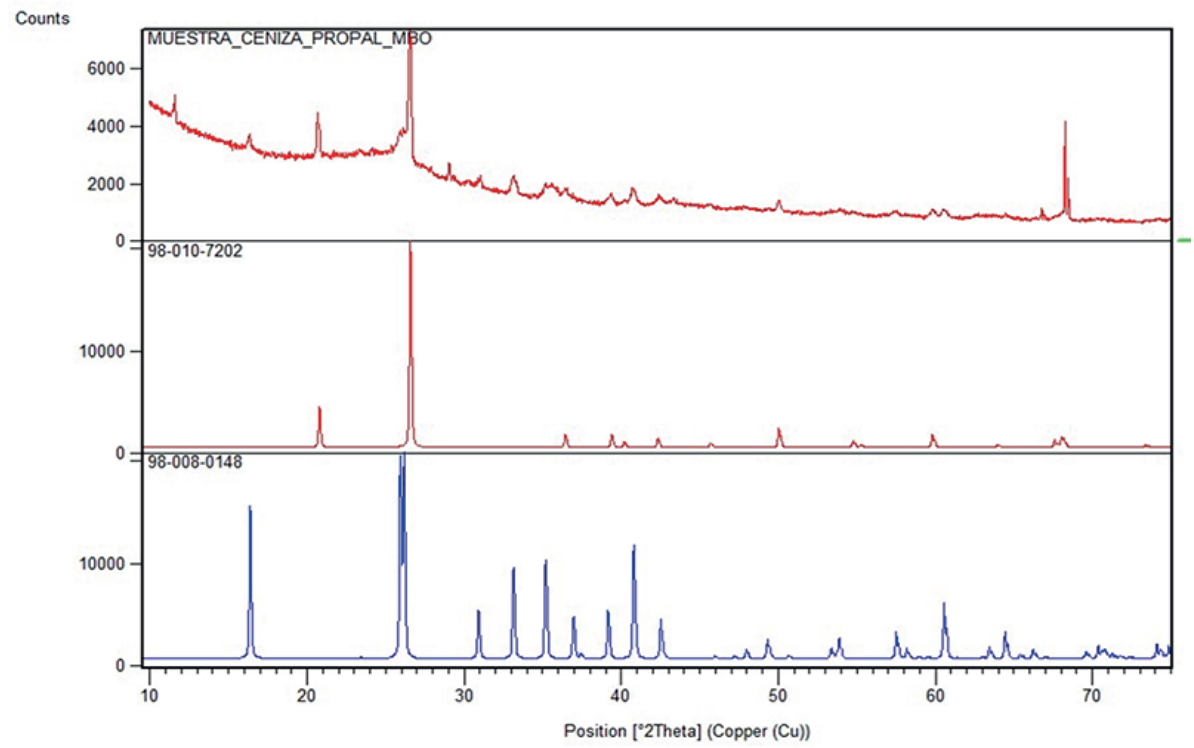

Figura 3. Difractograma y fases de la muestra de ceniza

Fuente: Informe del Laboratorio de Difracción de Rayos X de la Universidad del Valle, tomado de Ballesteros (2016).

Tabla 3. Absorción total y densidad de los especímenes.

\begin{tabular}{|l|l|l|l|l|l|}
\hline Propiedad & Testigo & $\mathbf{1 0} \%$ ceniza & $\mathbf{2 0} \%$ ceniza & $\mathbf{3 0} \%$ ceniza & $\mathbf{4 0} \%$ ceniza \\
\hline Absorción de agua $(\%)$ & $6,7 \%$ & $7,64 \%$ & $8,94 \%$ & $8,18 \%$ & $9,30 \%$ \\
\hline Densidad $\left(\mathrm{kg} / \mathrm{m}^{3}\right)$ & 2,211 & 2,156 & 2,107 & 2,088 & 2,080 \\
\hline
\end{tabular}

Fuente: basado de Ballesteros (2016).

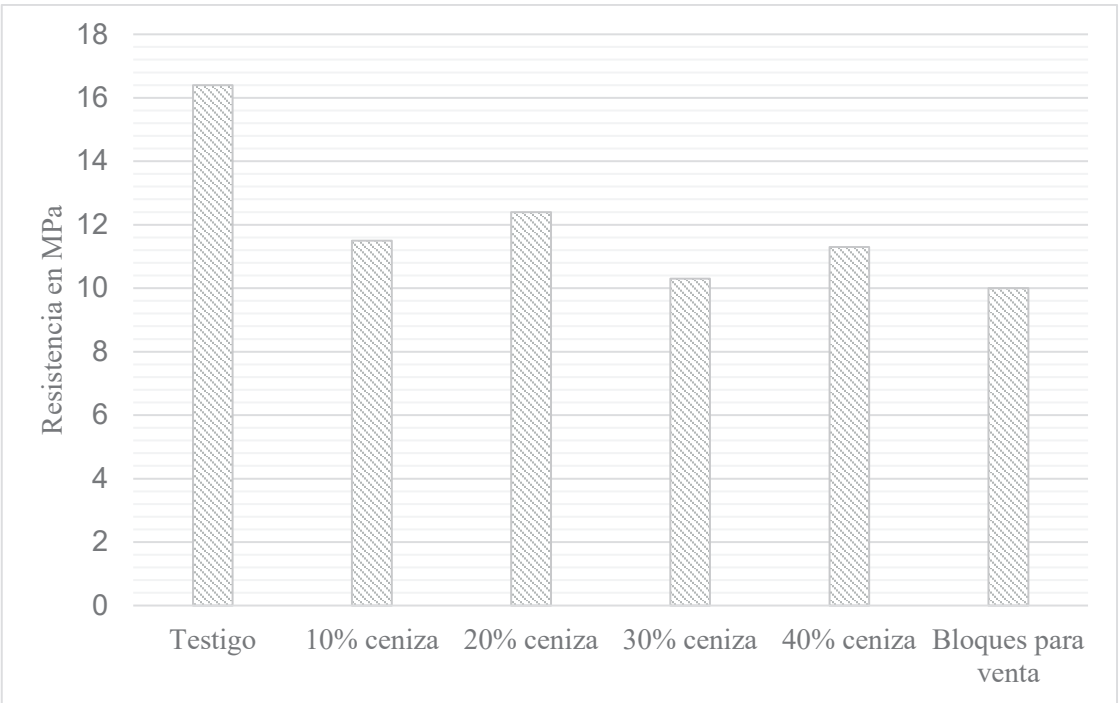

Figura 4. Resistencias promedio a compresión de los bloques.

Fuente: basado de Ballesteros (2016). 
Tabla 4. Resultados de resistencia a compresión.

\begin{tabular}{|c|c|c|c|}
\hline Espécimen & $\mathbf{M P a}$ & $\begin{array}{c}\text { Desviación } \\
\text { estándar }\end{array}$ & Varianza \\
\hline Testigo & 16,4 & 2,4 & 5,8 \\
\hline $10 \%$ ceniza & 11,5 & 2,4 & 5,6 \\
\hline $20 \%$ ceniza & 12,4 & 1,4 & 1,9 \\
\hline $30 \%$ ceniza & 10,3 & 1,6 & 2,4 \\
\hline $40 \%$ ceniza & 11,3 & 1,6 & 2,5 \\
\hline
\end{tabular}

Fuente: basado de Ballesteros (2016).

El comportamiento mecánico de los bloques modificados ensayados demostró que puede usarse la ceniza como reemplazo del cemento $40 \%$, por cuanto en todos los casos la resistencia del bloque fue superior a la resistencia del bloque estándar.

Al instante de la captura termográfica, la temperatura fue de $37,5^{\circ} \mathrm{C}$, con una humedad del $52 \%$. En general, la respuesta térmica de los bloques reveló que todos tuvieron la capacidad de absorber calor y que, en la medida en que se incrementó la proporción de ceniza, la capacidad de absorción también aumentó. Sin embargo, los huecos propios de la estructura de los bloques favorecen el paso del aire lo que hace que se concentre la temperatura hacia el centro del espécimen $y$, finalmente, logra que ocurra un descenso de temperatura hacia la parte superior donde nuevamente se presenta circulación de aire, como se observa en la respuesta térmica de los bloques (ver figuras $5 a$ a $5 \mathrm{e}$ ). Para el caso del bloque testigo ( $\sin$ ceniza, figura $5 a$ ), en el área demarcada (Ar1) se observa que la temperatura varía entre 37,6 y $42{ }^{\circ} \mathrm{C}$, mientras que en la zona central del bloque se mantiene una temperatura de $39,2{ }^{\circ} \mathrm{C}$. El piso de arcilla vitrificada se encuentra a una temperatura promedio de $40,7^{\circ} \mathrm{C}$; mientras que la zona sobre la cual se proyecta la sombra del bloque muestra los valores más bajos de temperatura $\left(34,3^{\circ} \mathrm{C}\right)$.

En cuanto al bloque con adición del $10 \%$ de ceniza (ver figura $5 b$ ), este presentó una variación de temperatura entre 36,9 y $46,2^{\circ} \mathrm{C}$, con un valor de temperatura para la zona central del bloque de $41,3^{\circ} \mathrm{C}$. El piso en arcilla vitrificada, al momento de la toma de las imágenes, se encontraba a $41{ }^{\circ} \mathrm{C}$ de temperatura, mientras que la zona bajo la sombra estaba a $34,3^{\circ} \mathrm{C}$. Al igual que en el caso del testigo, existe una circulación de aire causada por la ubicación del espécimen sobre el bloque de apoyo; aun así, la temperatura aumentó aproximadamente $1^{\circ} \mathrm{C}$ frente a la temperatura del testigo (ver figura $5 b$ ).

Con relación al bloque con $20 \%$ de sustitución de ceniza, la temperatura registrada osciló entre 38,1 y $46,2{ }^{\circ} \mathrm{C}$, con una temperatura al centro de 41,6
${ }^{\circ} \mathrm{C}$ (figura 5c). Por su parte, los bloques fabricados con $30 \%$ de sustitución con ceniza muestran temperaturas que varían entre 37,7 y $45,9^{\circ} \mathrm{C}$, con una temperatura al centro de $42,3^{\circ} \mathrm{C}$.

Finalmente, en el bloque preparado con $40 \%$ de ceniza, las temperaturas oscilaron entre 37,9 y 46,8 ${ }^{\circ} \mathrm{C}$, con una temperatura al centro de $42,8^{\circ} \mathrm{C}$ (figura $5 e), 3,6^{\circ} \mathrm{C}$ por encima del valor reportado en el bloque testigo, mostrando una tendencia ascendente, lo que sugiere que la adición de ceniza reduce la capacidad de refracción del concreto aumentado las temperaturas.

De las figuras 6a a 6 e se puede observar la variación térmica de los mampuestos y la máxima temperatura promedio hacia el centro, situación que se puede resumir de la siguiente forma:

- $\quad 39,8{ }^{\circ} \mathrm{C}$ en los bloques testigos (figura 6a).

- $\quad 41,6{ }^{\circ} \mathrm{C}$ en bloques preparados con $10 \%$ de ceniza (figura $6 b$ ).

- $\quad 42,2^{\circ} \mathrm{C}$ en aquellos bloques con $20 \%$ de ceniza (figura 6c).

- $\quad 41,8^{\circ} \mathrm{C}$ en los que fueron preparados con $30 \%$ de ceniza (figura $6 \mathrm{~d}$ ).

- $\quad 42,4{ }^{\circ} \mathrm{C}$ en los bloques en cuya fabricación se sustituyó el $40 \%$ de cemento por ceniza (figura $6 e)$.

En este estudio se tomaron los valores de temperatura de bloques individuales; sin embargo, es importante tener presente que el comportamiento termográfico se debe hacer en un muro de manera que sea posible estimar la radiación térmica absorbida por unidad de tiempo y unidad de superficie para definir, a nivel constructivo, si se requiere el uso de aislantes térmicos en aquellas zonas en donde se concentra la temperatura en el muro. Con esta base, se recomienda continuar los estudios experimentales para corroborar el compartimiento térmico identificado en los bloques, analizando el fenómeno en muros o muretes. Con relación a la adición de la ceniza proveniente de la combustión del carbón en el proceso de producción del papel los resultados de las pruebas a compresión confirman que esta ceniza podría ser empleada para la fabricación de bloques de concreto, incluso en sustituciones hasta del $40 \%$ de ceniza por cemento, tal y como lo sugieren otros estudios como el de Bouzoubaâ et al. (2011). Otros niveles de sustitución de cemento por cenizas también son atractivos (Asi y Abdullah, 2005; Topcu et al., 2008; Nuruddin et al., 2011; Chousidis et al., 2015; Rebeiz y Craft, 2002). 


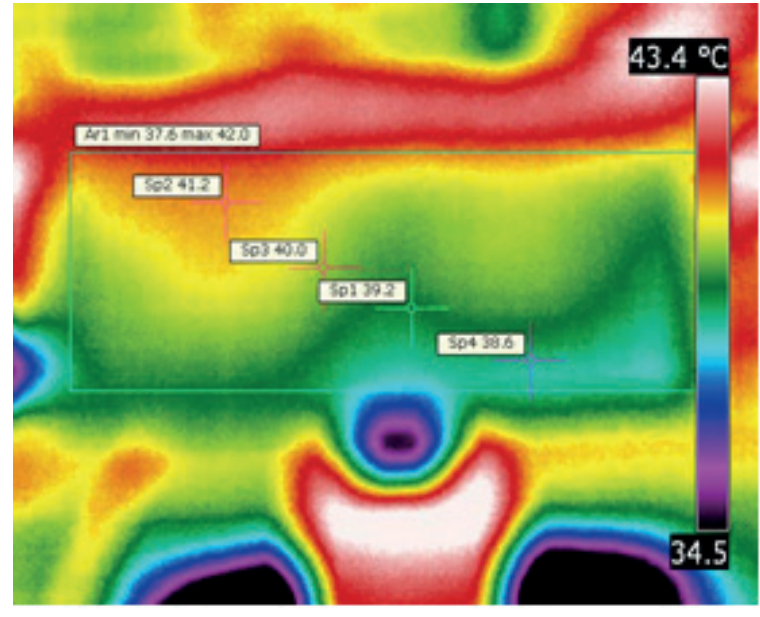

a) Bloque testigo.

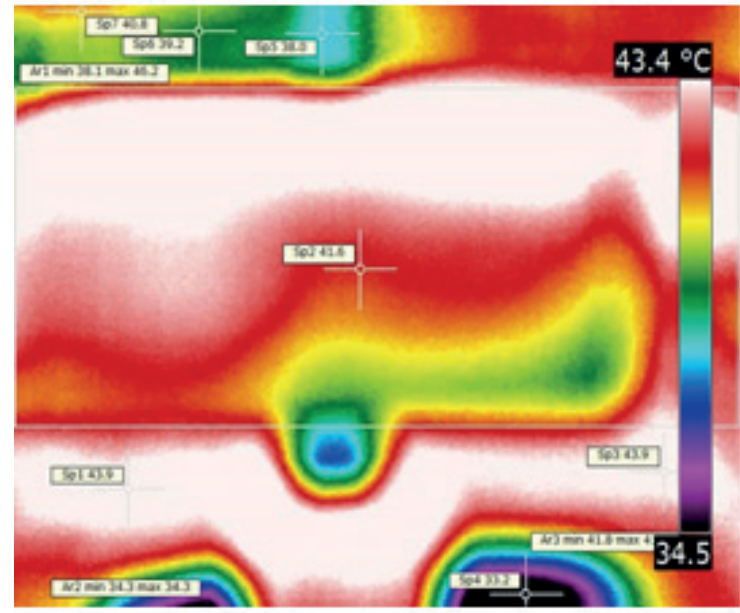

c) Bloque con adición de $20 \%$ de ceniza

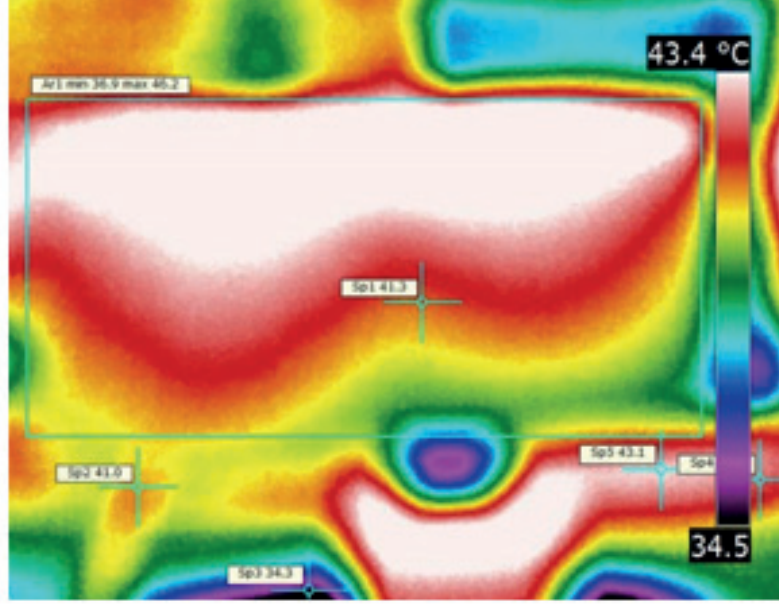

b) Bloque con adición de $10 \%$ de ceniza.

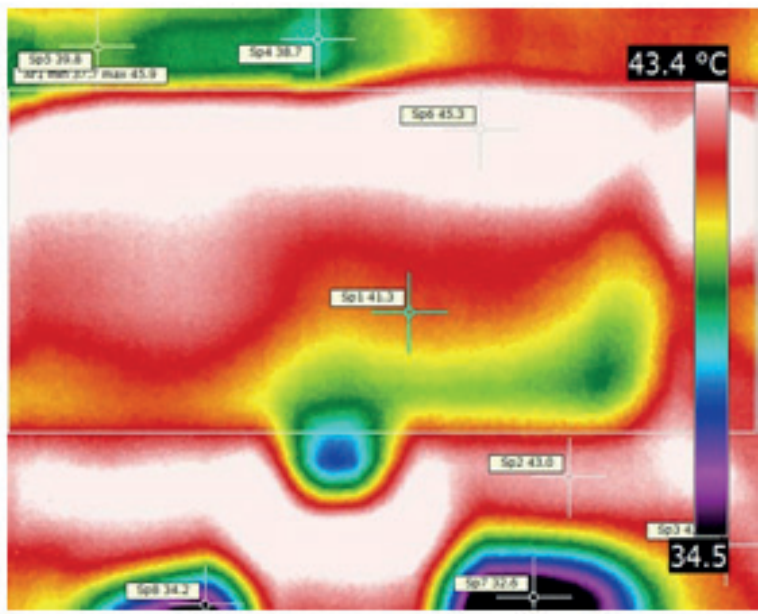

d) Bloque con adición de $30 \%$ de ceniza.

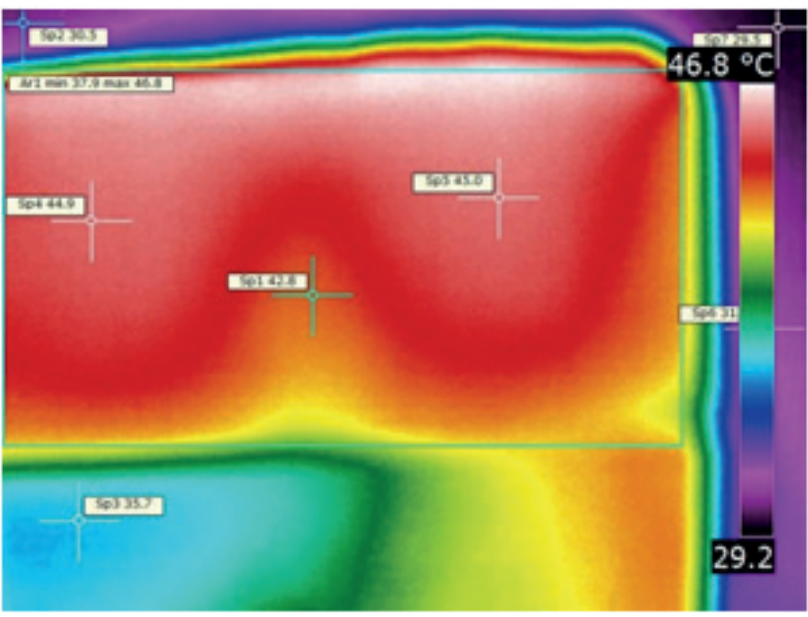

e) Bloque con adición de $40 \%$ de ceniza.

Figura 5. Variaciones de temperatura para diferentes adiciones de ceniza.

Fuente: basado de Ballesteros (2016). 


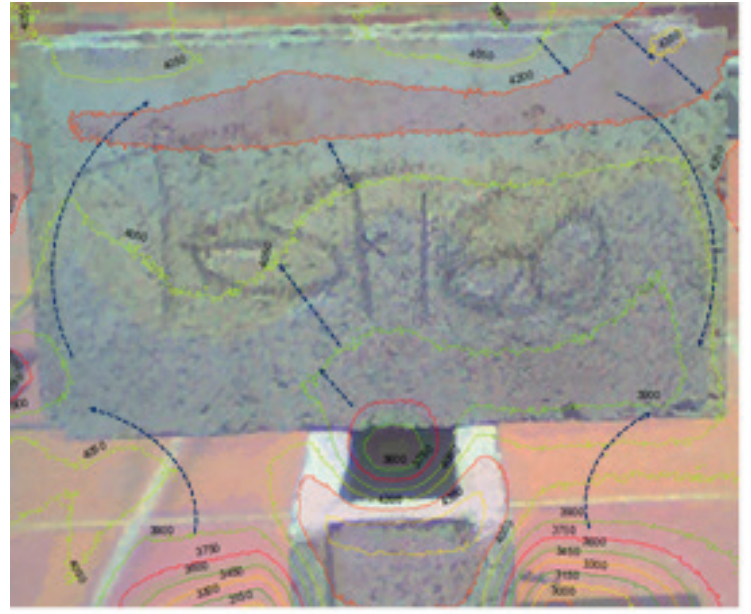

a) Máx. temperatura hacia el centro $40,5^{\circ} \mathrm{C}$.

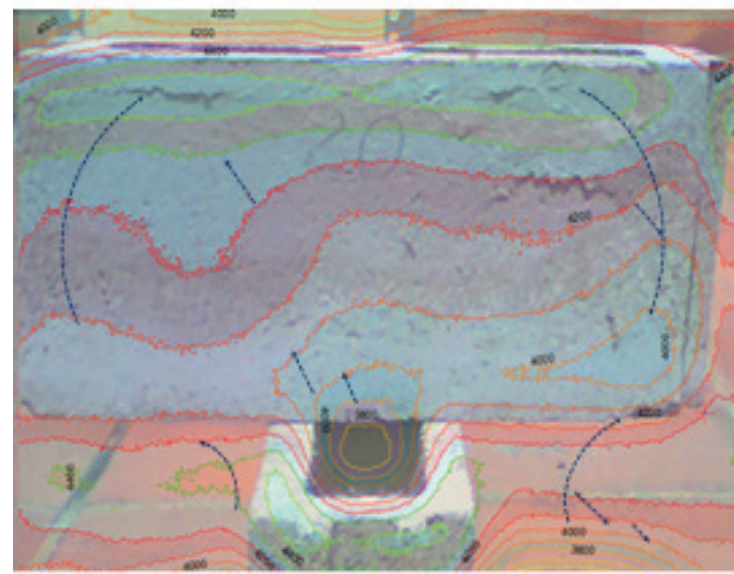

c) Máx. temperatura hacia el centro $42^{\circ} \mathrm{C}$.

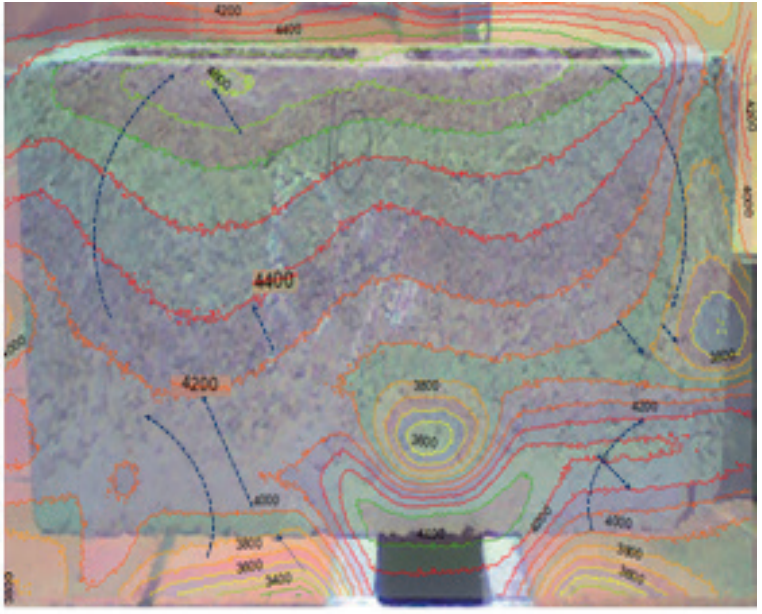

b) Máx. temperatura hacia el centro $44^{\circ} \mathrm{C}$.

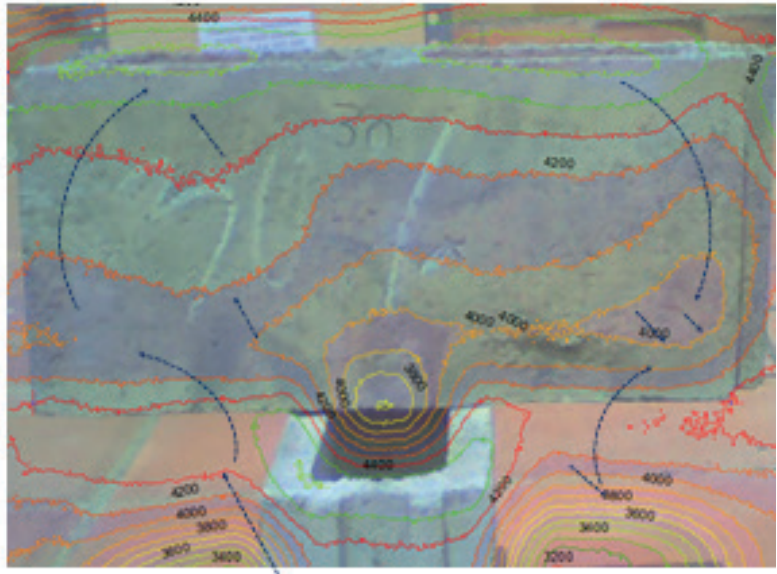

d) Máx. temperatura hacia el centro $42^{\circ} \mathrm{C}$.

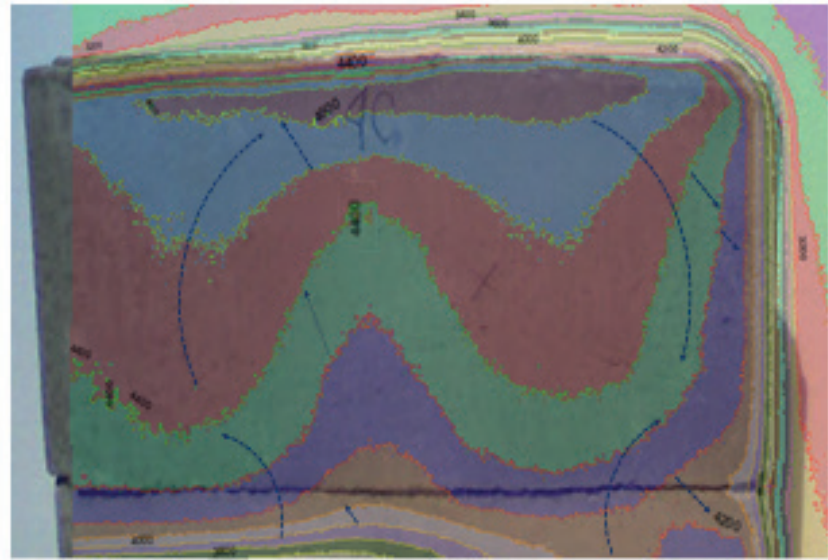

e) Máx. temperatura hacia el centro $44^{\circ} \mathrm{C}$.

Figura 6. Representación de curvas de temperatura para diferentes adiciones de ceniza. Fuente: basado de Ballesteros (2016). 


\section{CONCLUSIONES}

El progreso de la economía exige, en ocasiones, un consumo acelerado de recursos y materias primas, con el consecuente problema ambiental de la generación de residuos industriales. En este contexto, existe la posibilidad de hacer alianzas entre las empresas privadas, universidades y centros de investigación de modo que se estudien soluciones a problemas productivos relacionados con el medio ambiente y la fabricación de nuevos materiales. Por ejemplo, en este estudio se vinculó a una empresa productora de papel con una productora de bloques de concreto para estudiar la factibilidad de fabricar bloques de concreto adicionados con ceniza para uso no estructural. Se observó que los bloques de concreto pueden ser preparados con sustituciones hasta del $40 \%$ de ceniza sin afectar la resistencia que se ofrece al mercado en los bloques convencionales. Los estudios termográficos realizados en los bloques adicionados con cenizas industriales sugieren, de manera preliminar, que este material constructivo puede ser empleado para absorber el calor, lo que deberá corroborarse con investigaciones futuras.

\section{AGRADECIMIENTOS}

Se agradece la colaboración de la empresa Blokes S. A. y de la empresa Propal, así como de los estudiantes Juan Pablo Sánchez y Alejandro Torres de Ingeniería Civil de la Pontificia Universidad Javeriana Cali.

\section{REFERENCIAS BIBLIOGRÁFICAS}

[1] Asi, I. y Abdullah, A. (2005). Effect of Jordanian oil shale fly ash on asphalt mixes. Journal of Materials in Civil Engineering, 17(5), 553-559.

[2] Azevedo, A. A.; Martins, M. C. y Dal Molin, D. C. (2001). A study of the penetration of chloride in rice-husk ash concrete. International Concrete, 202, 379-396.

[3] Ballesteros, C. D. (2016). Ecobloques para aplicaciones no estructurales en el sector de la construcción. (Trabajo de maestría). Pontificia Universidad Javeriana, Cali, Colombia. Recuperado de http://vitela.javerianacali.edu. co/handle/11522/7312.

[4] Bouzoubaâ, N.; Bilodeau, A.; Fournier, B.; Hooton, R. D.; Gagné, R. y Jolin, M. (2011). Deicing salt scaling resistance of concrete incorporating fly ash and (or) silica fume: laboratory and field sidewalk test data. The Canadian Journal of Civil Engineering,
38, 373-382. Recuperado de https://www. nrcresearchpress.com/doi/full/10.1139/L08067.

[5] Bui, D.; Hu, J. y Stroeven, P. (2005). Particle size effect on the strength of rice husk ash blended gap-graded portland cement concrete. Cement and Concrete Composites, 27(3), 357366.

[6] Buitrago, R. (2015). Comunicación personal en la planta de producción de Blokes S. A. Cali, Colombia: s. e.

[7] Cámara Colombiana de la Construcción (2014). Tendencias de la construcción: economía y coyuntura sectorial. Informe de investigación: Características del mercado laboral en el sector de la construcción. Bogotá, Colombia: Coordenada Urbana.

[8] Chousidis, N.; Rakanta, E.; loannou, I. y Batis, G. (2015). Mechanical properties and durability performance of reinforced concrete containing fly ash. Construction and Building Materials, 101, 810-817.

[9] Delgado, J.; Martínez, J. P.; Guzmán, A.; Delvasto, S.; Amigó, V. y Sánchez, E. (2015). Reemplazo del cuarzo por ceniza de tamo de arroz en la manufactura de gres porcelánico. Revista EIA, 12(23), 41-50. Recuperado de https://revistas.eia.edu.co/index.php/reveia/ article/view/719/671.

[10] Guía de la termografía infrarroja. Aplicaciones en ahorro y eficiencia energética (2011). Madrid, España: Dirección General de Industria, Energía y Minas de la Comunidad de Madrid / Fundación de la Energía de la Comunidad de Madrid. Recuperado de: http://www.madrid. org/bvirtual/BVCM015258.pdf.

[11] Fernandes, I. (2015). Blocos e Pavers: produção e controle de qualidade. Sexta edición. São Paulo, Brasil: Treino Assessoria e Treinamentos Empresariais Ltda.

[12] Giaccio, G.; Rodríguez, G. y Zerbino, R. (2007). Failure mechanism of normal and high-strength concrete with rice-husk ash. Cement Concrete Composites, 29(7), 566-574.

[13] Hossain, K. M. A. y Lachemi, M. (2010). Fresh, mechanical, and durability characteristics of self-consolidating concrete incorporating volcanic ash. Journal of Materials in Civil Engineering, 22(7), 651-657.

[14] Le, H. T. y Ludwig, H. M. (2016). Effect of rice husk ash and other mineral admixtures on 
properties of self-compacting high performance concrete. Materials and Design, 89, 156-166. Recuperado de https://www.infona.pl/resource/ bwmeta1.element.elsevier-64262915-e1813efd-ab26-310bf86be35d.

[15] Nuruddin, M. F.; Demie, S. y Shaf, N. (2011). Effect of mix composition on workability and compressive strength of self-compacting geopolymer concrete. The Canadian Journal of Civil Engineering, 38(11), 1196-1203.

[16] Rebeiz, K. y Craft, A. (2002). Polymer concrete using coal fly ash, Journal of Energy Engineering, 128(3), 62-73.

[17] Serrano, M. F.; Pérez, D. D.; Barajas, C. y Oquendo, D. (2012). Beneficios del uso de agregados no convencionales en mezclas de concreto. Cemento y Hormigón, (951), 46-51.

[18] Serrano, M. F.; Pérez, D. D.; Sarmiento, C. O. y Grammes, F. (2013). Evaluación de las propiedades mecánicas de morteros modificados con cenizas provenientes de la desorción térmica de aguas de la industria petrolera. Cemento y Hormigón, (956), 10-14.
[19] Slim, G.; Morales M.; Alrumaidhin, L.; Bridgman, P.; Gloor, J.; Hoff, S. y Odem, W. (2016). Optimization of polymer-amended fly ash and paper pulp millings mixture for alternative landfill liner. Procedia Engineering, 145, 312-318.

[20] Teixeira, E. R.; Mateus, R.; Camoes, A. F.; Bragança, L. y Branco, F. (2016). Comparative environmental life-cycle analysis of concretes using biomass and coal fly ashes as partial cement replacement material. Journal of Cleaner Production, 112(4), 2221-2230.

[21] Topcu, I. B.; Toprak, M. U. y Akdag, D. (2008). Determination of optimal microwave curing cycle for fly ash mortars. The Canadian Journal of Civil Engineering, 35(4), 349-357.

[22] Vidal, H. (2016). Comunicación personal. Cali, Colombia.

[23] Xu, Y.; Wong, Y. L.; Poon, C. S. y Anson, M. (2003). Influence of PFA on cracking of concrete and cement paste after exposure to high temperatures. Cement and Concrete Research, 33(12), 2009-2016. 


\title{
Thermographic response of non-structural concrete blocks made with industrial ash
}

\author{
Cristian David Ballesteros Giraldo ${ }^{1}$ \\ María Fernanda Serrano GuZmán ${ }^{2}$ \\ Diego DARÍo PÉrez RUIZ ${ }^{3}$
}

\section{ABSTRACT}

Ash is a waste product of different commercial activities. For the paper industry, the disposal of this residue which results from the combustion of the coal used in the production process, represents a problem. For this reason, its use in the production of concrete blocks has been evaluated. The objective of this study is to compare the thermographic response of industriallymanufactured, non-structural concrete blocks that have been prepared with this ash. Additionally, the mechanical behavior of the blocks is compared when the ash is replaced on a percentage basis with cement. Likewise, substitutions of $10,20,30$ and $40 \%$ of ash for cement were made for blocks that were prepared in accordance with quality control requirements of a concrete block plant. Specialized techniques such as X-ray diffraction and microscopy demonstrated the absence of toxic substances in the ash. The study revealed that thermal absorption capacity increases as more ash is used.

Keywords: Blocks; ash; industrial waste; thermal insulation; resistance.

\section{INTRODUCTION}

Construction is an economic sector that boosts different productive sectors and also demands the consumption of different raw materials, equipment and human resources (Cámara Colombiana de la Construcción y Coordenada Urbana, 2014). In general, this industry generates important revenues, but also produces a lot of waste. However, other industries, such as papermaking, also generate waste, like ash, whose production can reach up to two tons per month (Vidal, 2016). The excessive production of waste and the inconvenience caused by the lack of space for its disposal, as well as the need to maintain a sustainable consumption of raw materials, have encouraged studies on the effect of the addition of industrial waste, such as ash, in the production of cement or concrete. For this reason, the ash coming from different sources such as the incineration of rice husk, the thermal desorption of sludge from hydrocarbon extraction, the incineration of fuels at thermal power plants, among others, has been evaluated for alternative uses, obtaining mixed results.

For example, concrete mixtures including 5 to $20 \%$ of rice husk ash resulted in low permeability due to pore volume decrease, which improves the resistance of these mixtures to aggressive agents (Azevedo et al., 2001). Subsequently, in a study by Giaccio et al. (2007) $10 \%$ of cement was replaced by ash resulting in mixtures of high-strength concrete (80MPa) with good surface absorption behavior. Other studies report that dosages of $20 \%$ fly ash and $20 \%$ rice husk ash, after a 56 -day curing process, makes it possible to develop concrete with strength up to $130 \mathrm{MPa}$. (Le \& Ludwig, 2016). The ash from thermal desorption of sludges from oil locations processes can accelerate the initial setting time when added in percentages of less than $15 \%$, although a reduction in compressive strength up to $12 \%$ can be seen in relation to control mixtures (Serrano et al., 2013). On the other hand, mixtures with compressive strengths up to $125 \mathrm{MPa}$ have been

1 Master in Civil Engineering from the Pontificia Universidad Javeriana (Cali, Colombia). Currently working as an independent consultant. E-mail: davidballesteros1@hotmail.com

2 PhD. in Civil Engineering from University of Puerto Rico Mayaguez Campus. Currently working as Professor of Civil Engineering at Pontificia Universidad Javeriana Cali, Colombia. E-mail: maria.serrano@javerianacali.edu.co Orcid: http://orcid.org/0000-0002-7366-6597

$3 \mathrm{PhD}$. in Civil Engineering from University of Texas Arlington, United States. Currently working as Professor of Civil Engineering at Pontificia Universidad Javeriana Cali, Colombia. E-mail: ddperez@javerianacali.edu.co 
produced by adding pulverized fuel ash in percentages of 25 and $55 \%$ (Xu et al., 2003). By adding volcanic ash in percentages of $50 \%$, the mixtures have presented an average compressive strength of 15 MPa (Hossain \& Lachemi, 2010). Finally, regarding the ash from pulverized coal combustion in power plants, when it is added to replace $10 \%$ cement, it produces effective behaviors in the improvement of the mechanical properties of concrete (Asi \& Abdullah, 2005), reaching strengths in some cases up to $51 \mathrm{MPa}$ (Nuruddin et al., 2011), and furthermore improves the durability of the concrete exposed to saline environments (Chousidis et al., 2015).

As correctly stated by Topcu et al. (2008), the origin of the ash is varied, as is its use in the production of mortar and concrete (Teixeira et al., 2016) for the manufacture of non-structural concrete blocks such as porcelain stoneware tile (Delgado et al., 2015), in modified concrete and mortars (Serrano et al., 2012; Serrano et al., 2013), as well as its use as material in geotechnical and soil solutions and other agricultural uses (Slim et al., 2016) or in terraces (Bouzoubaâ et al., 2011). Likewise, as ash is used as a replacement for cement, the mixtures produced are cheaper and most studies have shown an increase in the strength and durability of the mixtures, and their use is recommended in high performance concrete (Bui et al., 2005).

In this context, the objective of this study is to present the thermographic response when paper industry waste is included in the manufacture of concrete blocks according to the production system of a Colombian company and to compare the mechanical compression response of the blocks produced against the standards of the company. This type of masonry opens the door for a partnership between concrete block and paper producers, since the results of this investigation indicate that the addition of ash did not affect the compressive strength of the block produced and improved its response to temperature changes.

\section{METHODOLOGY}

The analysis of the thermal response of blocks was done in two stages: it began with analysis of the ash supplied by the paper mill and ended with the analysis of the thermal response of the blocks.

\section{Microscopic characterization of ash}

A scanning electron microscope (JEOL model JSM 6490 LV) was used and chemical composition changes were reviewed via the images using an acceleration voltage of $15 \mathrm{kV}$. Additionally, chemi- cal microanalyses of the samples were performed on various inspection points or areas. For this, an Oxford Instrument model INCAPentaFETx3 EDS probe was used.

\section{X-ray diffraction test}

For the X-ray analysis, a high-resolution device (X'Pert-MRD PANalytical) was used and the data was analyzed using Xpert Data software. The sample was characterized by comparing the reflections obtained with those reported in the PDF2-Release-2009 International Center for Diffraction Data (ICDD) database for pure and polycrystalline materials.

\section{Manufacturing procedure for the blocks studied}

Each block manufacturer employs a particular dosage for block preparation. This dosage can vary among block producing companies, according to Fernandes (2005), since the materials are conditioned in the same production line, depending on the conditions of the material, state of the machinery and other factors that affect the quality of the pieces (Buitrago, 2015). Therefore, in this investigation an industrialized procedure was used for the production of thirty typical blocks of the Valle del Cauca (Colombia), whose dosage was adjusted to prepare blocks replacing $10,20,30$ and $40 \%$ cement for ash, according to the proportions indicated in Table 1. Additionally, the particle size distribution test for fine aggregates was carried out following the ASTM C-136 standard.

The production stages of the blocks studied are summarized as follows:

- The condition of machinery and raw materials was reviewed.

- $\quad$ The texture required for non-structural blocks was established.

- The cohesion of the mixture was checked to ensure that the block remained intact during its manufacture.

- The block was compacted and it was verified that it had a hard consistency (with the "finger test") and that, when pouring water on the surface, it did not pass freely through the material. The blocks were stored in the curing room.

- Quality control was carried out in accordance with Instituto Colombiano de Normas Técnicas [Colombian Institute of Technical Standards] technical quality standards NTC 4205-1, 4205-2 
and 4205-3 in order to determine weight, total absorption, density, net volume and net area.

- $\quad$ The compression test on the dry units was carried out, considering the direction in which the blocks would be put into service; in this case, with vertical gaps (NTC-ISO 7500-1 standard for class 1).

\section{Thermographic tests}

The thermal imager should maintain the same focal length $(0.70 \mathrm{~m})$, perpendicular to the face of the monitored specimen and keep the same height of the tripod $(0.30 \mathrm{~m})$. This imager highlights temperature variations and hot spots in real time and makes it possible to combine visible light image with a thermal image from the object of study (Dirección General de Industria, Energía y Minas de la Comunidad de Madrid y la Fundación de la Energía de la Comunidad de Madrid, 2011). The temperature response of blocks previously exposed to solar radiation for three days was evaluated. Additionally, with a temperature and humidity sensor, readings of these parameters were taken in order to incorporate this data for the calibration and data processing with the imager.

\section{RESULTS}

Sand and quarry dust had a fineness modulus of 2.43 and 2.78 , respectively, so both materials can be classified as sand with high fines content. As for the ash, the test performed with the scanning electron microscope enabled the identification of its chemical composition (see Table 2), according to results obtained in the seven points indicated in Figure 1, which shows the microscopic detail of the ash with fifty times surface magnification. The results of the chemical composition indicate the presence of carbon, oxygen and silicon, with average concentration values of $48.2,24.5$ and $11.9 \%$, respectively, suggesting that the ash could be used in the production of concrete.

The results of the $\mathrm{X}$-ray diffraction analysis show the phases (codes 98-010-7202 and 98-008-0148) present in the material (see Figures 2 and 3 ) and identified silicon oxide in hexagonal crystal structure and aluminum silicon oxide in orthorhombic crystal structure in the database. These results suggested the possibility of using this ash in the manufacture of blocks.

Table 1. Test matrix.

\begin{tabular}{|l|c|c|c|c|c|c|}
\hline Block & Cement $(\mathbf{k g})$ & Dust $\left(\mathbf{m}^{\mathbf{3}}\right)$ & Sand $\left(\mathbf{m}^{3}\right)$ & Water I & Additive $(\mathbf{c c})$ & Ash $(\mathbf{k g})$ \\
\hline Control & 50 & 0.231 & 0.099 & 18.00 & 200 & 0.00 \\
\hline $10 \%$ replacement & 45 & 0.231 & 0.099 & 20.00 & 200 & 5.00 \\
\hline $20 \%$ replacement & 40 & 0.231 & 0.099 & 22.00 & 200 & 10.00 \\
\hline $30 \%$ replacement & 35 & 0.231 & 0.099 & 25.50 & 200 & 15.00 \\
\hline $40 \%$ replacement & 30 & 0.231 & 0.099 & 25.50 & 200 & 20.00 \\
\hline
\end{tabular}

Source: Based on Ballesteros (2016).

Table 2. Chemical composition of ash (percentage).

\begin{tabular}{|l|c|c|c|c|c|c|c|c|c|}
\hline Spectrum & $\mathbf{C}$ & $\mathbf{O}$ & $\mathbf{N a}$ & $\mathbf{A l}$ & $\mathbf{S i}$ & $\mathbf{K}$ & $\mathbf{C a}$ & $\mathbf{T i}$ & $\mathbf{F e}$ \\
\hline 1 & 11.64 & 36.75 & 2.87 & 19.96 & 23.27 & & & 2.23 & 3.29 \\
\hline 2 & 85.81 & 11.01 & & 1.57 & 1.62 & & & & \\
\hline 3 & 63.55 & 19.20 & & 3.09 & 11.85 & & 2.31 & & \\
\hline 4 & 25.73 & 39.15 & 1.72 & 3.92 & 28.33 & 1.14 & & & \\
\hline 5 & 15.48 & 16.96 & & 5.31 & 8.84 & & 0.88 & & 52.53 \\
\hline 6 & 76.96 & 18.34 & & 2.39 & 2.31 & & & & \\
\hline 7 & 58.20 & 30.20 & & 4.70 & 6.90 & & & & \\
\hline Average & 48.20 & 24.52 & 2.30 & 5.85 & 11.87 & 1.14 & 1.60 & 2.23 & 27.91 \\
\hline Standard deviation & 30.20 & 10.80 & 0.80 & 6.40 & 10.30 & NA & 1.00 & NA & 34.80 \\
\hline Coefficient of variation & 62.70 & 44.00 & 34.80 & 109.40 & 86.80 & NA & 62.50 & NA & 124.70 \\
\hline
\end{tabular}

Source: Based on Ballesteros (2016). 
Regarding absorption and density of concrete blocks, according to Colombian Technical Standard NTC 4076, the blocks including ash complied with the provisions for concrete with densities of 2000 $\mathrm{kg} / \mathrm{m}^{3}$ or more, which must have a total absorption percentage not exceeding 12\% (see Table 3).

At 28 days, the ash-free specimens as well as the specimens with diverse ash additions showed strength greater than or equal to $10 \mathrm{MPa}$, which is the strength value of the non-structural blocks offered in the market (see Figure 4).

In general, the mechanical strength of the prepared blocks exceeded the mechanical strength of the blocks normally offered in the market (Table 4).

The mechanical behavior of the modified blocks tested showed that ash can be used as a $40 \%$ cement replacement, since in all cases the strength

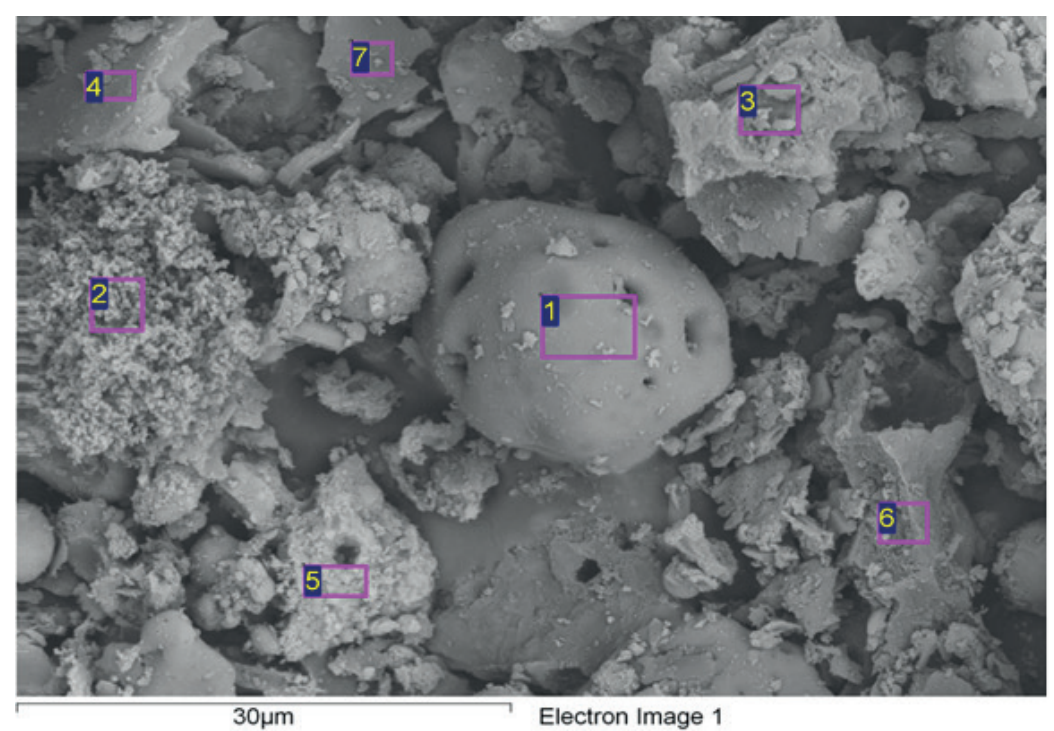

Figure 1. Microscopic details of the ash.

Source: Report of the X-Ray Diffraction Laboratory at the Universidad del Valle, taken from Ballesteros (2016).

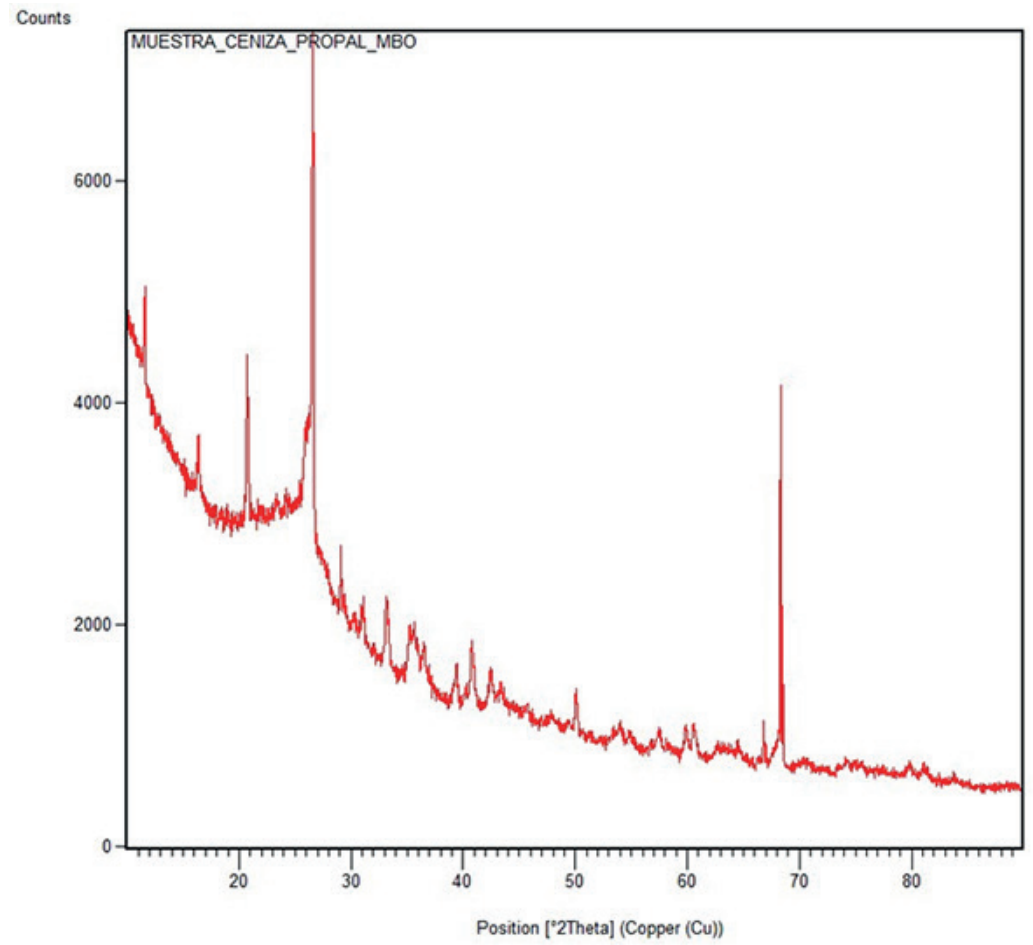

Figure 2. Diffractogram of the ash sample.

Source: Report of the X-Ray Diffraction Laboratory at Universidad del Valle, taken from Ballesteros (2016). 
of the block was greater than the strength of the standard block.

At the time of thermographic capture, the temperature was $37.5{ }^{\circ} \mathrm{C}$, with a humidity of $52 \%$. In general, the thermal response of the blocks revealed that all of them could absorb heat and that, as the proportion of ash increased, the absorption capacity increased too. However, the characteristic gaps of the block's structure favor the passage of air, which causes that the temperature concentrates towards the center of the specimen and, fina-

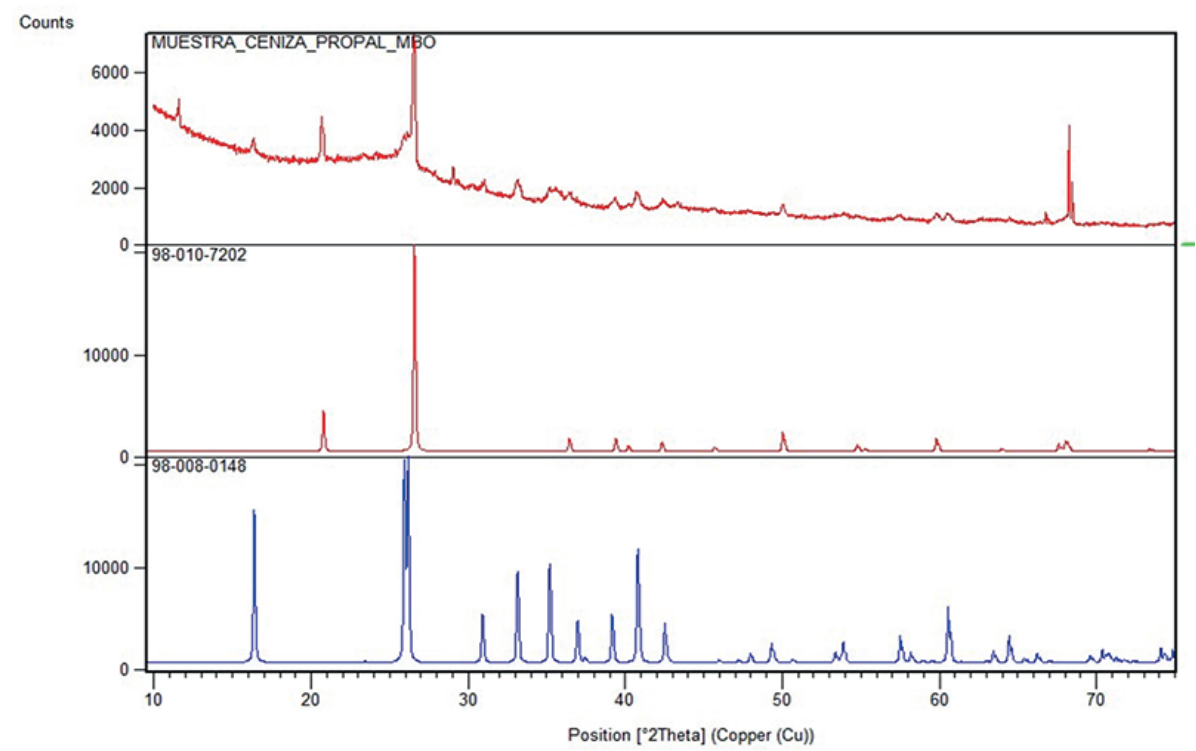

Figure 3. Diffractogram and phases of the ash sample.

Source: Report of the X-Ray Diffraction Laboratory at Universidad del Valle, taken from Ballesteros (2016).

Table 3. Total absorption and density of specimens.

\begin{tabular}{|l|c|c|c|c|c|}
\hline Property & Control & $\mathbf{1 0} \%$ ash & $\mathbf{2 0} \%$ ash & $\mathbf{3 0 \%}$ ash & $\mathbf{4 0 \%}$ ash \\
\hline Water absorption $(\%)$ & $6.7 \%$ & $7.64 \%$ & $8.94 \%$ & $8.18 \%$ & $9.30 \%$ \\
\hline Density $\left(\mathrm{kg} / \mathrm{m}^{3}\right)$ & 2.211 & 2.156 & 2.107 & 2.088 & 2.080 \\
\hline
\end{tabular}

Source: Based on Ballesteros (2016).

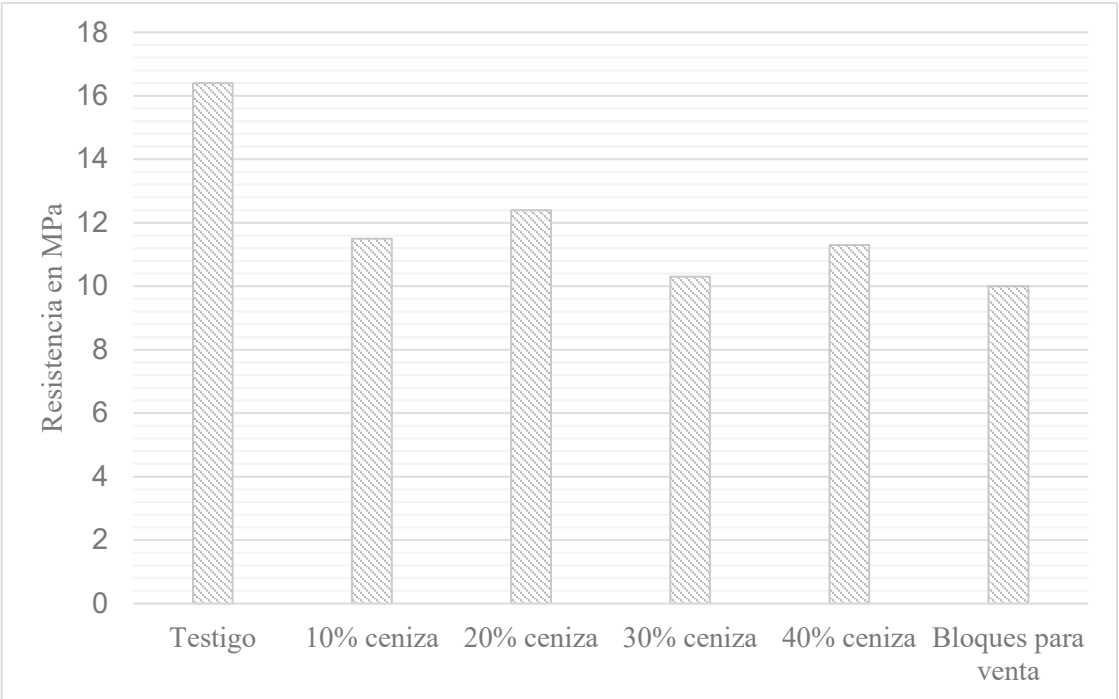

Figure 4. Average compressive strengths of the blocks Source: Based on Ballesteros (2016). 
lly, causes a temperature drop at the upper part, where circulation of air occurs again, as seen in the thermal response of the blocks (see figures $5 a$ to $5 e$ ). In the delimited area (Ar1) of the control block (without ash, Figure $5 \mathrm{a}$ ), it is observed that the temperature varies between 37.6 and $42{ }^{\circ} \mathrm{C}$, while in the central area the temperature is maintained at $39.2^{\circ} \mathrm{C}$. The vitrified clay floor is at an average temperature of $40.7^{\circ} \mathrm{C}$; while the area on which the block's shadow is cast shows the lowest temperature values $\left(34.3^{\circ} \mathrm{C}\right)$.

Table 4. Compressive strength results.

\begin{tabular}{|c|c|c|c|}
\hline Specimen & MPa & $\begin{array}{c}\text { Standard } \\
\text { deviation }\end{array}$ & Variance \\
\hline Control & 16.4 & 2.4 & 5.8 \\
\hline $10 \%$ ash & 11.5 & 2.4 & 5.6 \\
\hline $20 \%$ ash & 12.4 & 1.4 & 1.9 \\
\hline $30 \%$ ash & 10.3 & 1.6 & 2.4 \\
\hline $40 \%$ ash & 11.3 & 1.6 & 2.5 \\
\hline
\end{tabular}

As for the block with the addition of $10 \%$ ash (see Figure $5 \mathrm{~b}$ ), its temperature varied between 36.9 and $46.2^{\circ} \mathrm{C}$, with a temperature value of $41.3^{\circ} \mathrm{C}$ for the central area. The vitrified clay floor was at $41{ }^{\circ} \mathrm{C}$ while the area under the shade was $34.3^{\circ} \mathrm{C}$. As in the case of the control block, there is an airflow due to the location of the specimen on the support block; even so, the temperature increased by approximately $1{ }^{\circ} \mathrm{C}$ compared to the temperature of the control block (see Figure 5b).Source: Based on Ballesteros (2016).

In relation to the block with $20 \%$ ash replacement, the recorded temperature fluctuated between 38.1 and $46.2{ }^{\circ} \mathrm{C}$, with $41.6{ }^{\circ} \mathrm{C}$ at the center (Figure 5c). Meanwhile, the blocks manufactured with $30 \%$ ash replacement show temperatures that vary between 37.7 and $45.9^{\circ} \mathrm{C}$, with $42.3^{\circ} \mathrm{C}$ at the center.

Finally, in the block prepared with $40 \%$ ash, temperatures fluctuated between 37.9 and $46.8{ }^{\circ} \mathrm{C}$, with 42.8 ${ }^{\circ} \mathrm{C}$ at the center (Figure $5 \mathrm{e}$ ), $3.6{ }^{\circ} \mathrm{C}$ above the value reported in the control block, showing an upward trend, which suggests that the addition of ash reduces refractive capacity, increasing temperature.

Figures $6 a$ to $6 e$ show the thermal variation of the rough blocks and the maximum average temperature towards the center, which can be summarized as follows:

- $\quad 39.8^{\circ} \mathrm{C}$ in the control blocks (Figure 6a).

- $41.6^{\circ} \mathrm{C}$ in blocks prepared with $10 \%$ ash (Figure $6 b)$.
- $\quad 42.2{ }^{\circ} \mathrm{C}$ in blocks prepared with $20 \%$ ash (Figure $6 \mathrm{c})$.

- $\quad 41.8{ }^{\circ} \mathrm{C}$ in blocks prepared with $30 \%$ ash (Figure $6 \mathrm{~d})$.

- $\quad 42.4{ }^{\circ} \mathrm{C}$ in blocks prepared with $40 \%$ ash (Figure 6e).

In this study, temperature values of individual blocks were taken; however, it is important to keep in mind that thermographic behavior must be assessed on a wall so that it is possible to estimate the thermal radiation absorbed per unit of time and surface unit in order to define, at construction level, whether the use of thermal insulators is required in those areas of the wall where the temperature is concentrated. Based on this, it is recommended to continue experimental studies to corroborate the thermal behavior identified in the blocks, analyzing this phenomenon in walls or dwarf walls. Regarding the addition of ash from coal combustion during the paper production process, compressive tests results confirm that this ash could be used for the manufacture of concrete blocks, even replacing up to $40 \%$ of cement by ash, as suggested by other studies such as Bouzoubaâ et al. (2011). Other levels of cement replacement by ashes are also attractive (Asi \& Abdullah, 2005; Topcu et al., 2008; Nuruddin et al., 2011; Chousidis et al., 2015; Rebeiz \& Craft, 2002).

\section{CONCLUSIONS}

Economic progress sometimes requires an accelerated consumption of resources and raw materials, resulting in environmental problems due to industrial waste generation. In this context, making alliances between private companies, universities and research centers is possible, so that solutions to production problems related to the environment and the manufacture of new materials are studied. For example, in this study, a paper mill was linked to a concrete block producer to study the feasibility of manufacturing concrete blocks for non-structural use adding ash. It was observed that concrete blocks can be prepared with replacements up to $40 \%$ ash without affecting the strength offered by conventional blocks to the market. Thermographic studies carried out on the blocks with added industrial ash suggest, on a preliminary basis, that this construction material can be employed to absorb heat, which should be corroborated with future studies.

\section{ACKNOWLEDGEMENTS}

The collaboration of Blokes S. A. company and Propal company, as well as Civil Engineering students 


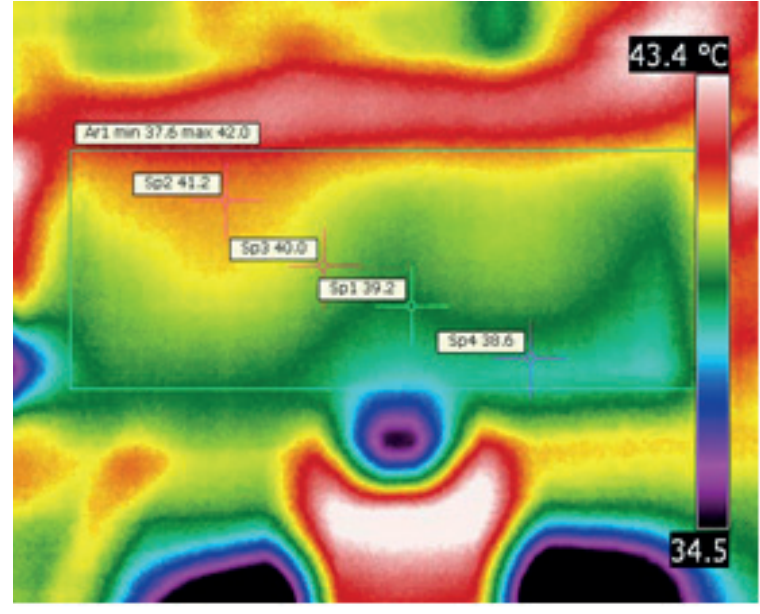

a) Control block.

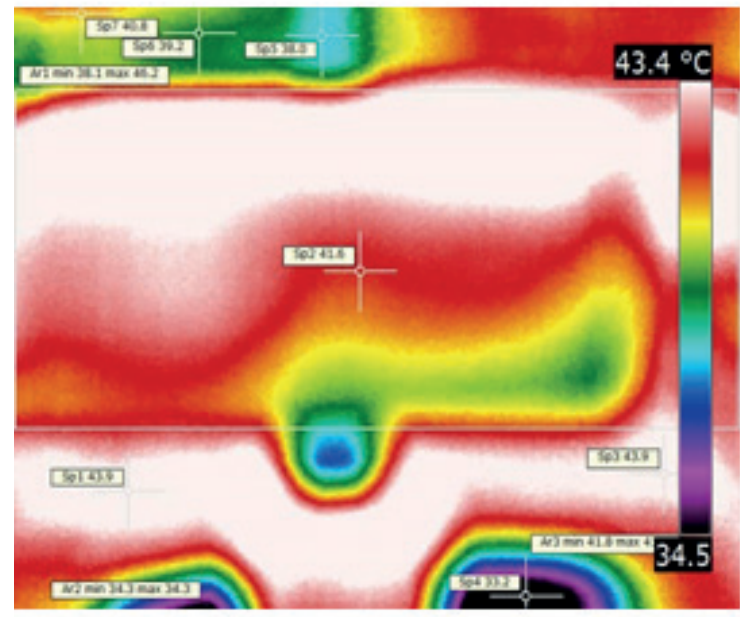

c) Block with $20 \%$ ash.

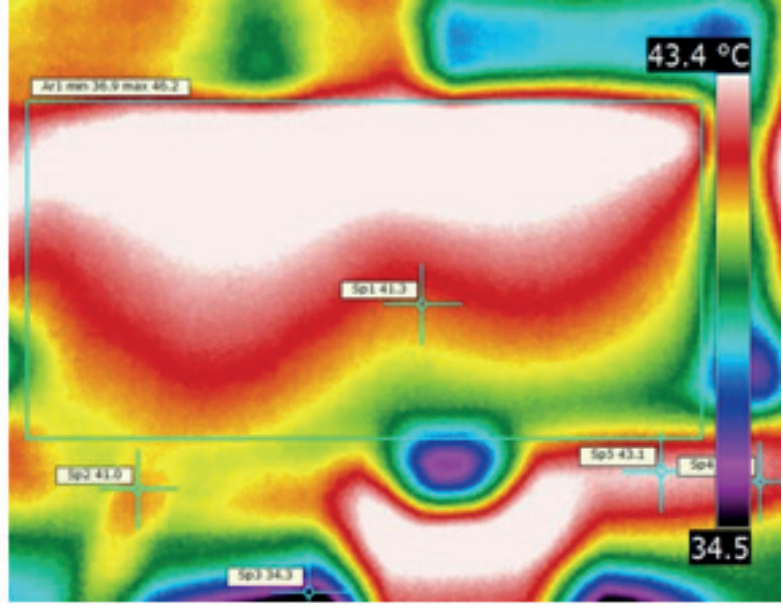

b) Block with $10 \%$ ash.

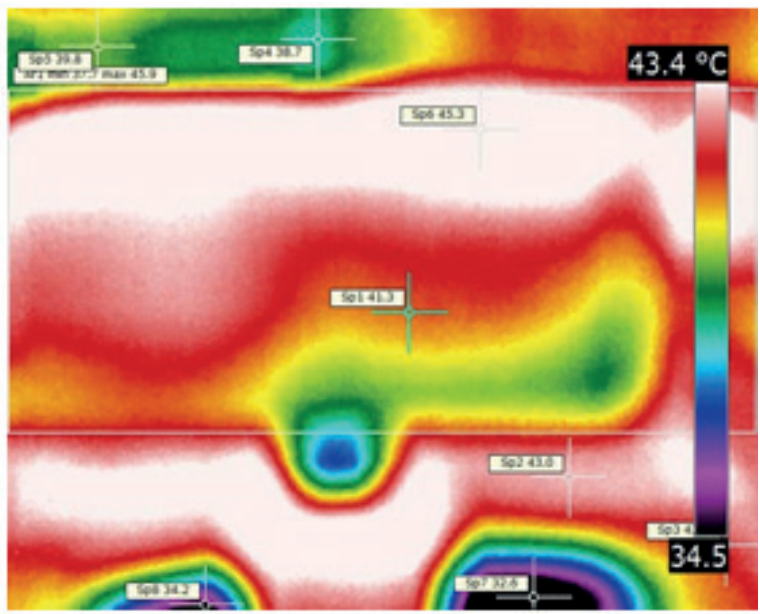

d) Block with $30 \%$ ash.

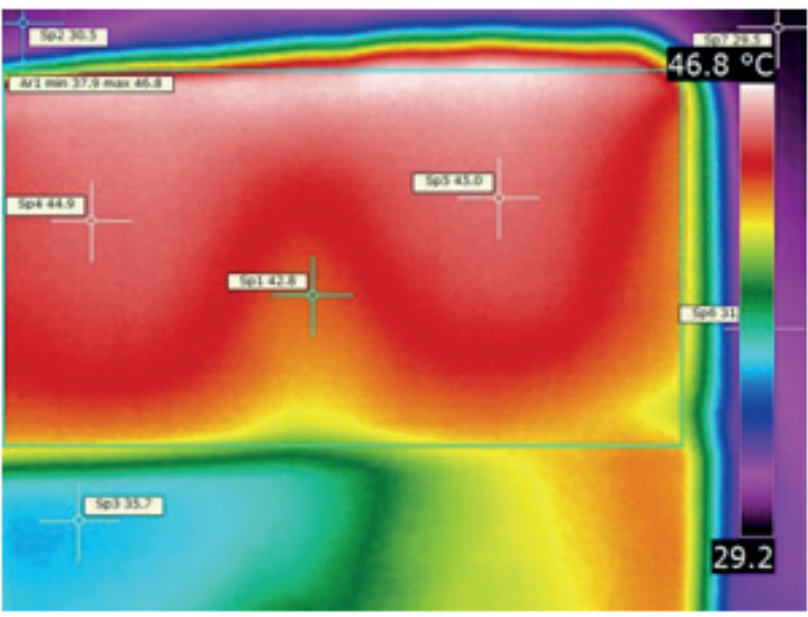

e) Block with $40 \%$ ash.

Figure 5. Temperature variations for different ash additions.

Source: Based on Ballesteros (2016). 


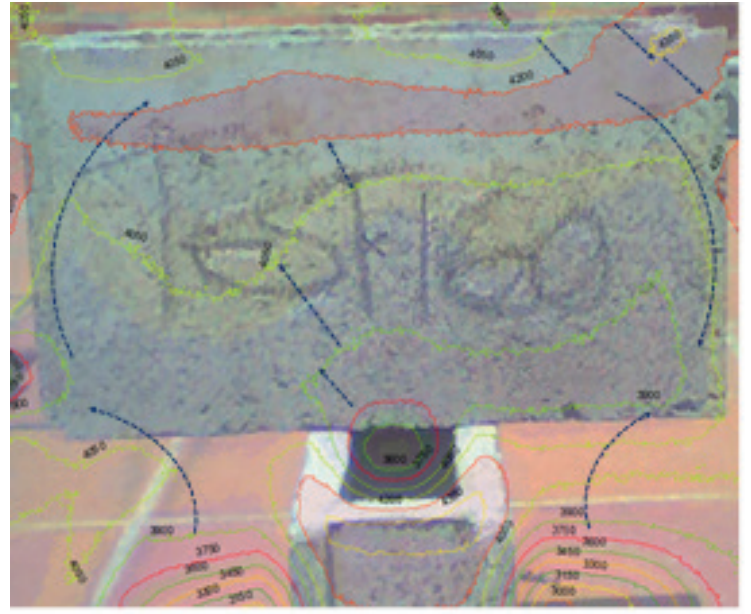

a) Max. temperature towards the center: $40.5^{\circ} \mathrm{C}$.

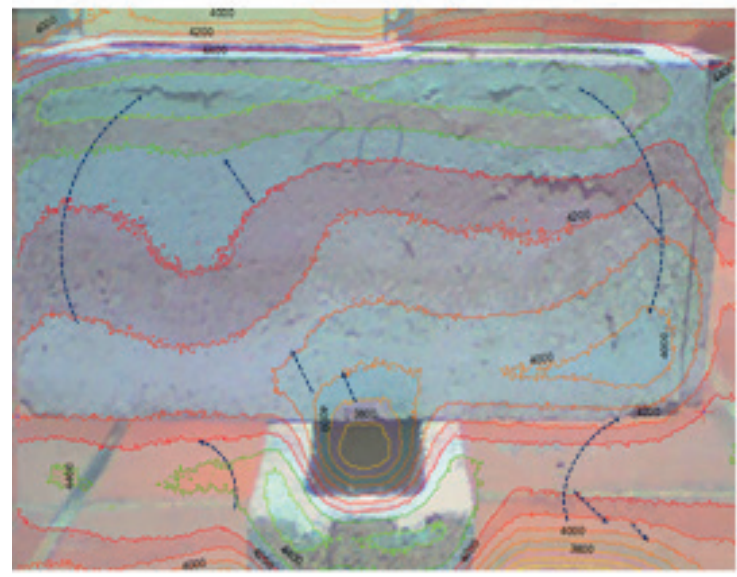

c) Max. temperature towards the center: $42^{\circ} \mathrm{C}$.

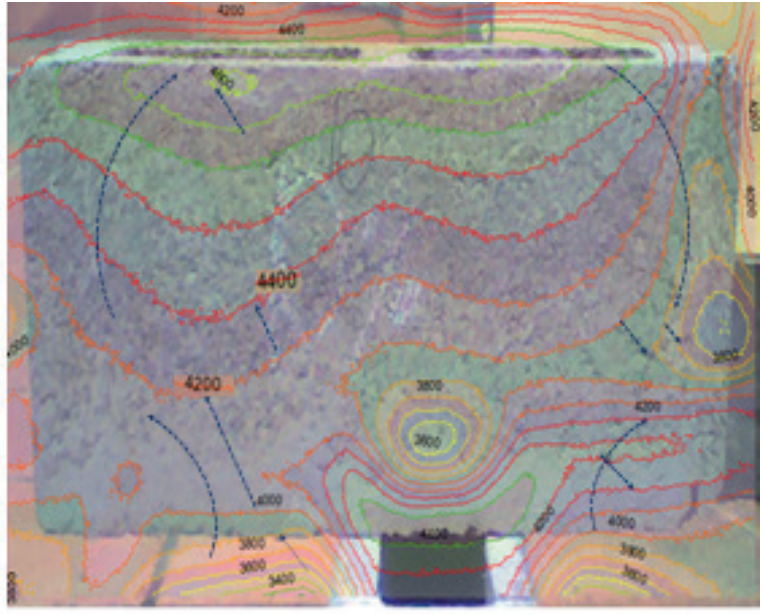

b) Max. temperature towards the center: $44^{\circ} \mathrm{C}$.

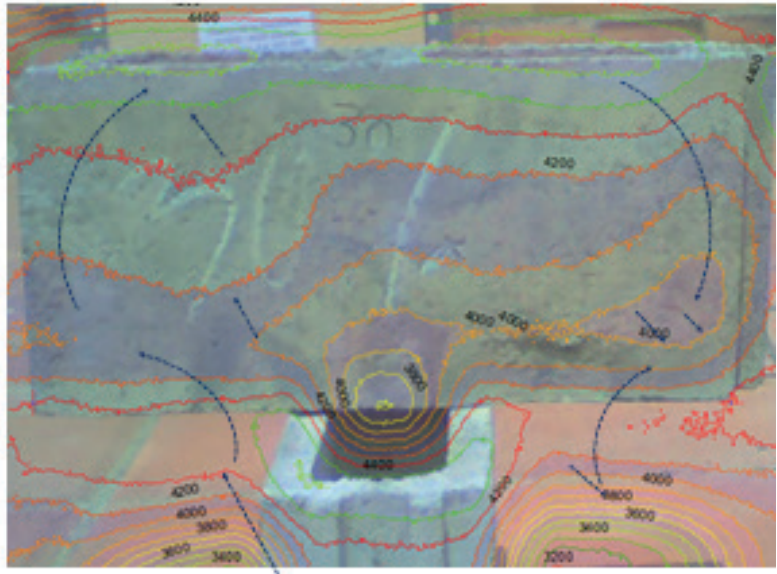

d) Max. temperature towards the center: $42^{\circ} \mathrm{C}$.

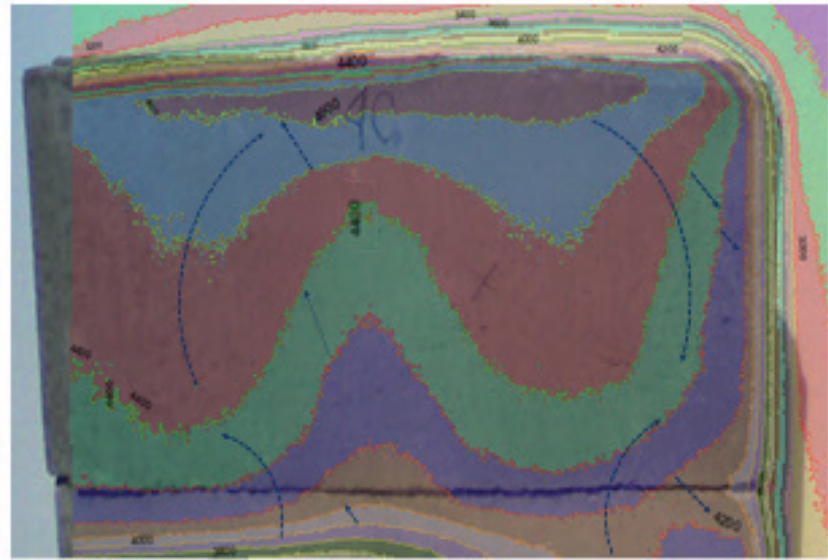

e) Max. temperature towards the center: $44^{\circ} \mathrm{C}$.

Figure 6. Representation of temperature curves for different ash additions.

Source: Based on Ballesteros (2016). 
of the Pontificia Universidad Javeriana Cali, Juan Pablo Sánchez and Alejandro Torres, are gratefully acknowledged.

\section{REFERENCES}

[1] Asi, I. \& Abdullah, A. (2005). Effect of Jordanian oil shale fly ash on asphalt mixes. Journal of Materials in Civil Engineering, 17(5), 553-559.

[2] Azevedo, A. A.; Martins, M. C. \& Dal Molin, D. C. (2001). A study of the penetration of chloride in rice-husk ash concrete. International Concrete, 202, 379-396.

[3] Ballesteros, C. D. (2016). Ecobloques para aplicaciones no estructurales en el sector de la construcción. (Master's thesis). Pontificia Universidad Javeriana, Cali, Colombia. Retrieved from http://vitela.javerianacali.edu. co/handle/11522/7312.

[4] Bouzoubaâ, N.; Bilodeau, A.; Fournier, B.; Hooton, R. D.; Gagné, R. \& Jolin, M. (2011). Deicing salt scaling resistance of concrete incorporating fly ash and (or) silica fume: laboratory and field sidewalk test data. The Canadian Journal of Civil Engineering, 38, 373-382. Retrieved from https://www. nrcresearchpress.com/doi/full/10.1139/L08-067.

[5] Bui, D.; Hu, J. \& Stroeven, P. (2005). Particle size effect on the strength of rice husk ash blended gap-graded portland cement concrete. Cement and Concrete Composites, 27(3), 357-366.

[6] Buitrago, R. (2015). Comunicación personal en la planta de producción de Blokes S. A. Cali, Colombia: s. e.

[7] Cámara Colombiana de la Construcción (2014). Tendencias de la construcción: economía y coyuntura sectorial. Informe de investigación: Características del mercado laboral en el sector de la construcción. Bogota, Colombia: Coordenada Urbana.

[8] Chousidis, N.; Rakanta, E.; loannou, I. \& Batis, G. (2015). Mechanical properties and durability performance of reinforced concrete containing fly ash. Construction and Building Materials, 101, 810-817.

[9] Delgado, J.; Martínez, J. P.; Guzmán, A.; Delvasto, S.; Amigó, V. \& Sánchez, E. (2015). Reemplazo del cuarzo por ceniza de tamo de arroz en la manufactura de gres porcelánico. Revista EIA, 12(23), 41-50. Retrieved from https://revistas.eia.edu.co/index.php/reveia/ article/view/719/671.
[10] Guía de la termografía infrarroja. Aplicaciones en ahorro y eficiencia energética (2011). Madrid, Spain: Dirección General de Industria, Energía y Minas de la Comunidad de Madrid / Fundación de la Energía de la Comunidad de Madrid. Retrieved from http://www.madrid.org/ bvirtual/BVCM015258.pdf.

[11] Fernandes, I. (2015). Blocos e Pavers: produção e controle de qualidade. Sexta edición. São Paulo, Brazil: Treino Assessoria e Treinamentos Empresariais Ltda.

[12] Giaccio, G.; Rodríguez, G. \& Zerbino, R. (2007). Failure mechanism of normal and high-strength concrete with rice-husk ash. Cement Concrete Composites, 29(7), 566-574.

[13] Hossain, K. M. A. \& Lachemi, M. (2010). Fresh, mechanical, and durability characteristics of self-consolidating concrete incorporating volcanic ash. Journal of Materials in Civil Engineering, 22(7), 651-657.

[14] Le, H. T. \& Ludwig, H. M. (2016). Effect of rice husk ash and other mineral admixtures on properties of self-compacting high performance concrete. Materials and Design, 89, 156-166. Retrieved from https://www.infona.pl/resource/ bwmeta1.element.elsevier-64262915-e1813efd-ab26-310bf86be35d.

[15] Nuruddin, M. F.; Demie, S. \& Shaf, N. (2011). Effect of mix composition on workability and compressive strength of self-compacting geopolymer concrete. The Canadian Journal of Civil Engineering, 38(11), 1196-1203.

[16] Rebeiz, K. \& Craft, A. (2002). Polymer concrete using coal fly ash, Journal of Energy Engineering, 128(3), 62-73.

[17] Serrano, M. F.; Pérez, D. D.; Barajas, C. \& Oquendo, D. (2012). Beneficios del uso de agregados no convencionales en mezclas de concreto. Cemento y Hormigón, (951), 46-51.

[18] Serrano, M. F.; Pérez, D. D.; Sarmiento, C. O. \& Grammes, F. (2013). Evaluación de las propiedades mecánicas de morteros modificados con cenizas provenientes de la desorción térmica de aguas de la industria petrolera. Cemento y Hormigón, (956), 10-14.

[19] Slim, G.; Morales M.; Alrumaidhin, L.; Bridgman, P.; Gloor, J.; Hoff, S. \& Odem, W. (2016). Optimization of polymer-amended fly ash and paper pulp millings mixture for alternative landfill liner. Procedia Engineering, 145, 312-318. 
[20] Teixeira, E. R.; Mateus, R.; Camoes, A. F.; Bragança, L. \& Branco, F. (2016). Comparative environmental life-cycle analysis of concretes using biomass and coal fly ashes as partial cement replacement material. Journal of Cleaner Production, 112(4), 2221-2230.

[21] Topcu, I. B.; Toprak, M. U. \& Akdag, D. (2008). Determination of optimal microwave curing cycle for fly ash mortars. The Canadian Journal of Civil Engineering, 35(4), 349-357.
[22] Vidal, H. (2016). Comunicación personal. Cali, Colombia.

[23] Xu, Y.; Wong, Y. L.; Poon, C. S. \& Anson, M. (2003). Influence of PFA on cracking of concrete and cement paste after exposure to high temperatures. Cement and Concrete Research, 33(12), 2009-2016. 